\title{
Estimation of Average Local-to-Unity Roots in Heterogenous Panels
}

Erik Hjalmarsson

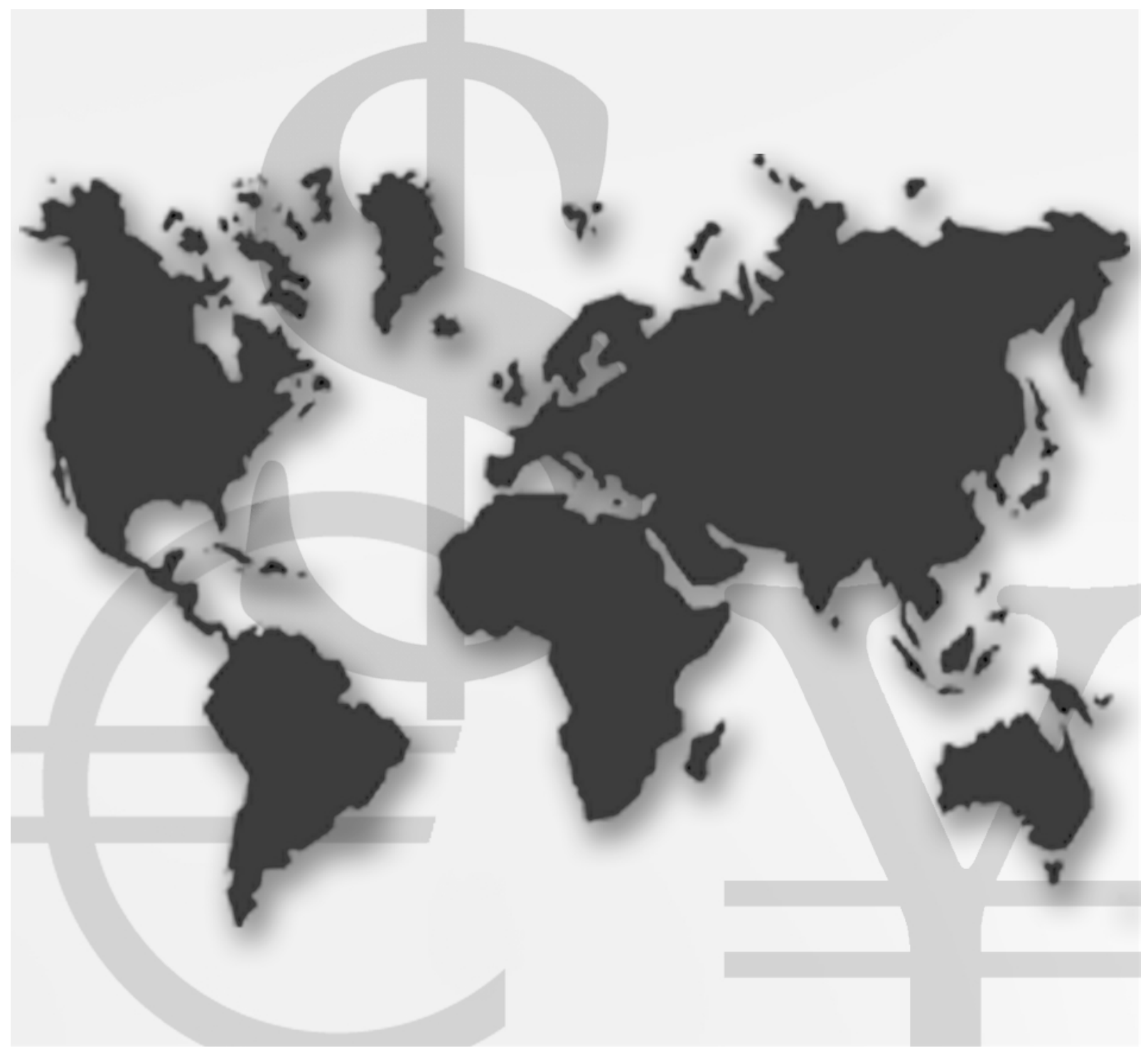

International Finance Discussion Papers

Board of Governors of the Federal Reserve System

Number 852

December 2005 


\title{
Board of Governors of the Federal Reserve System
}

\author{
International Finance Discussion Papers
}

Number 852

December 2005

\section{Estimation of Average Local-to-Unity Roots in Heterogenous Panels}

\author{
Erik Hjalmarsson
}

NOTE: International Finance Discussion Papers are preliminary materials circulated to stimulate discussion and critical comment. References in publications to International Finance Discussion Papers (other than an acknowledgment that the writer has had access to unpublished material) should be cleared with the author or authors. Recent IFDPs are available on the Web at www.federalreserve.gov/pubs/ifdp/. 



\title{
Estimation of Average Local-to-Unity Roots in Heterogenous Panels*
}

\author{
Erik Hjalmarsson ${ }^{\dagger}$ \\ Division of International Finance \\ Federal Reserve Board, Mail Stop 20, Washington, DC 20551, USA
}

December 2005

\begin{abstract}
This paper considers the estimation of average autoregressive roots-near-unity in panels where the time-series have heterogenous local-to-unity parameters. The pooled estimator is shown to have a potentially severe bias and a robust median based procedure is proposed instead. This median estimator has a small asymptotic bias that can be eliminated almost completely by a bias correction procedure. The asymptotic normality of the estimator is proved. The methods proposed in the paper provide a useful way of summarizing the persistence in a panel data set, as well as a complement to more traditional panel unit root tests.
\end{abstract}

JEL classification: C22, C23.

Keywords: Local-to-unity; Panel data; Pooled regression; Median estimation; Bias correction.

\footnotetext{
*I am grateful to Peter Phillips and Don Andrews for providing much useful advice. Other helpful comments have also been provided by Lennart Hjalmarsson, Randi Hjalmarsson, Catalin Starica, and Jonathan Wright as well as seminar participants at the European meeting of the Econometric Society in Madrid, 2004, and the econometrics seminar at Göteborg University.

${ }^{\dagger}$ Tel.: +1-202-452-2436; fax: +1-202-263-4850; email: erik.hjalmarsson@frb.gov. The views presented in this paper are solely those of the author and do not represent those of the Federal Reserve Board or its staff.
} 



\section{Introduction}

Few concepts have had such an impact on recent econometric practice as unit roots. The modern asymptotic theory developed for integrated processes clearly shows that a failure to account for the order of integration of the data can lead to flawed inference. However, many economic time-series exhibit a nearly persistent behavior with the largest auto-regressive root close to one, which often makes it difficult to distinguish between stationary and non-stationary series in practice. This has led to the increasing popularity of so called nearly integrated processes as a modelling device; rather than maintaining a strict dichotomy between integrated and non-integrated time-series, the largest auto-regressive root is treated as being local-to-unity which allows for a smoother transition between the stationary and non-stationary worlds.

Originally, nearly intergrated processes were mainly used for theoretical excercises, such as evaluating the local power properties of unit-root tests (e.g. Phillips and Perron, 1988, and Elliot et al., 1996). Lately, however, they have also become increasingly popular in practical inference (e.g. Cavanagh et al., 1995, and Campbell and Yogo, 2003). Although the generalization from a standard unit-root environment to a near integrated environment provides more flexibility, it suffers from the drawback that the key characterstic parameter of such a model, the local-to-unity parameter, cannot be estimated in a time-series setting. ${ }^{1}$ However, as shown in a series of papers by Moon and Phillips (1999, 2000, and 2004), the local-to-unity parameter can be estimated using a panel of observations, when all of the time-series have identical local-to-unity parameters. In practice, the assumption that all of the time-series in the panel have an identical degree of persistence is obviously very restrictive. In this paper I, therefore analyze the estimation of local-to-unity roots in panels where the degree of persistence varies between the time-series.

The purpose of this paper is twofold. First, I consider the properties of the pooled estimator of localto-unity parameters proposed by Moon and Phillips (2000) in the case where the individual time-series possess differing degrees of persistence. Second, I propose a new estimator for the average local-tounity root in a panel, based on applying the median operator to extract the crucial cross-sectional information in the panel.

When there is no longer a common local-to-unity parameter in a panel, a desirable property of a panel based estimator would be that it consistently estimates the mean, or average, parameter value in

\footnotetext{
${ }^{1}$ Phillips et al. (1998) do provide a method of estimating local-to-unity roots from a single time-series using a block model. However, their specification of the local-to-unity model is somewhat different from the one typically adopted in the literature.
} 
the panel. As is shown, however, the pooled estimator of Moon and Phillips (2000) can be a severely biased estimator of the average parameter, even for relatively modest deviations from the case of identical local-to-unity roots.

The basic idea of the pooled estimator is that a consistent estimator can be obtained by taking the inconsistent OLS time-series estimator of the local-to-unity parameter and summing up over the crosssection in both the numerator and the denominator. Since this method fails when the local-to-unity parameters are no longer identical, I propose a more robust approach by applying the sample median estimator, rather than the sample mean, in both the numerator and denominator of the time-series estimator.

The bias and consistency properties of the resulting estimator cannot be analytically evaluated, but results based on numerical integration are straightforward to obtain. After a simple bias correction, the estimator is shown to be consistent in the case with identical local-to-unity parameters in the panel. More importantly, under the additional assumption that the local-to-unity parameters are normally distributed, it is shown that the estimator converges to a quantity that is very close to the average local-to-unity parameter, regardless of the variance in the distribution of the local-to-unity parameters. That is, in the case of identical near unit-roots in the panel, the estimator is consistent and it is very close to consistent in the case of non-identical roots. The bias in the non-identical case is small, and likely to be negligible compared to the variance of the estimates in any finite sample. The asymptotic normality of the estimator is also shown, as well as the estimation of standard errors and confidence intervals. Monte Carlo simulations support these results and also indicate that the estimator works well in cases where the local-to-unity parameters are not normally distributed.

The results developed in this paper are useful along several dimensions. First, they highlight the potential hazards of applying estimators of near-unit roots designed for the case of identical local-tounity roots throughout the panel, when there is in fact a possibility that the roots are non-identical. Second, it is shown how to estimate the average near unit-root in a panel data set. This can be useful both as a characterization of the data in itself, as well as a starting point for further empirical analysis. It also provides a complement to panel unit-root tests, which have recently become very popular. ${ }^{2}$ The methods in this paper provide a simple diagnostic addition to these tests by estimating the average auto-regressive root in the panel. Since confidence intervals for this average root can also be computed, further conclusions can also be obtained. For instance, a confidence interval that is strictly below zero

\footnotetext{
${ }^{2}$ See for instance Quah (1994), Maddala and Wu (1999), Choi (2001), Levin et al. (2002), Moon and Perron (2003), and Moon et al. (2003).
} 
reveals that the average root is significantly less than zero; hence, some of the actual roots in the panel must also be negative.

The rest of the paper is organized as follows. Section 2 details the setup and main assumptions and Section 3 derives the bias properties of the pooled estimator. The main results of the paper are developed in Section 4, where the asymptotic properties of the median based estimator are derived, and Section 5 concludes. All proofs and details of the numerical calculations are found in the Appendix.

A word on notation, $\Rightarrow$ denotes weak convergence of the associated probability measures and $\rightarrow p$ denotes convergence in probability. I write $(n, T \rightarrow \infty)$ when $n$ and $T$ go to infinity simultaneously and $(T, n \rightarrow \infty)_{\text {seq }}$ when $T$ goes to infinity first while keeping $n$ fixed, and then letting $n$ go to infinity.

\section{Model and assumptions}

Let the data generating process for each individual time series, $z_{i, t}$, satisfy

$$
\begin{aligned}
z_{i, t} & =\beta_{i, 0}+y_{i, t}, \quad i=1, \ldots, n ; t=1, \ldots, T \\
y_{i, t} & =a_{i} y_{i, t-1}+\epsilon_{i, t}, \quad a_{i}=1+\frac{c_{i}}{T}=1+\frac{c+\eta_{i}}{T},
\end{aligned}
$$

where $y_{i, t}$ is a near integrated process with local-to-unity parameter $c_{i}=c+\eta_{i}$. The focus of interest in this paper is the estimation of the average, or mean, local-to-unity parameter $c$.

The following assumptions on the error processes and the local to unity parameters, $c_{i}$, will be useful.

Assumption $1 \epsilon_{i, t}$ are linear processes satisfying

(a) $\epsilon_{i, t}=D_{i}(L) u_{i, t}=\sum_{j=0}^{\infty} D_{i, j} u_{i, t-j}, \quad \sum_{j=0}^{\infty} j^{b}\left|D_{i, j}\right|<\infty$ for some $b \geq 1, \quad\left|D_{i}(1)\right| \neq 0$.

(b) $u_{i, t}$ are iid across $i$ and over $t$ with $E\left(u_{i, t}\right)=0, E\left(u_{i, t}^{2}\right)=1$, and finite fourth order moments.

Let $D_{i}=D_{i}(1), \Omega_{i}=D_{i}^{2}$, and $\Lambda_{i}=\sum_{j=1}^{\infty} D_{i, 0} D_{i, j}$, so that $\Omega_{i}$ and $\Lambda_{i}$ specify the long-run variance and the one-sided long-run covariance matrix, respectively, of $\epsilon_{i, t}$.

Assumption 2 The random variables $c_{i}, i=1, \ldots, n$ are normally distributed with mean $c$ and variance $\sigma_{c}^{2}$.

Under Assumption 1, it is well known that as $T \rightarrow \infty, y_{i,[T r]} / \sqrt{T} \Rightarrow D_{i} J_{i, c_{i}}(r)$, where $J_{i, c_{i}}(r)=$ $\int_{0}^{r} e^{(r-s) c_{i}} d W_{i}(s)$ and $W_{i}(r)$ is a standard Brownian motion (e.g. Phillips, 1987). 


\section{The bias properties of the pooled estimator}

I first show that the pooled estimator of $c$ does not work well when the $c_{i}$ are non-identical for all $i$. To keep the discussion as transparent as possible, consider the simple case where $\epsilon_{i, t}$ are $i i d\left(0, \sigma_{\epsilon}^{2}\right)$ across $i$ and $t$; the arguments presented could easily be modified to account for the general error processes in Assumption 1. Also, to keep the discussion short, only sequential limit arguments are presented.

Noting that $a_{i}=1+\frac{c_{i}}{T}=1+\frac{c+\eta_{i}}{T}=a+\frac{\eta_{i}}{T}$, I consider estimators of the form $\hat{c}=T(\hat{a}-1)$. The pooled estimator of $a$ is given by,

$$
\hat{a}=\frac{\sum_{i=1}^{n} \sum_{t=1}^{T} z_{i, t-1} z_{i, t}}{\sum_{i=1}^{n} \sum_{t=1}^{T} z_{i, t-1}^{2}},
$$

and the corresponding pooled estimator of $c$ is $\hat{c}=T(\hat{a}-1)$. By equation (2),

$$
\begin{aligned}
T(\hat{a}-a)= & {\left[\frac{1}{n} \sum_{i=1}^{n} \frac{1}{T^{2}} \sum_{t=1}^{T}\left(\beta_{i, 0}+y_{i, t-1}\right)^{2}\right]^{-1} } \\
& \times\left[\frac{1}{n} \sum_{i=1}^{n} \frac{1}{T} \sum_{t=1}^{T}\left[\left(\beta_{i, 0}+y_{i, t-1}\right)\left((1-a) \beta_{i, 0}+\epsilon_{i, t}+\frac{\eta_{i}}{T} y_{i, t-1}\right)\right]\right] \\
\Rightarrow & {\left[\frac{1}{n} \sum_{i=1}^{n} \int_{0}^{1} J_{i, c_{i}}(r)^{2} d r\right]^{-1} } \\
& {\left[\frac{1}{n} \sum_{i=1}^{n} \int_{0}^{1} J_{i, c_{i}}(r) d W_{i}(r)+\frac{1}{n} \sum_{i=1}^{n} \eta_{i} \int_{0}^{1} J_{i, c_{i}}(r)^{2} d r\right], }
\end{aligned}
$$

as $T \rightarrow \infty$. As shown below, both $E\left[\int_{0}^{1} J_{i, c_{i}}(r)^{2} d r\right]$ and $E\left[\eta_{i} \int_{0}^{1} J_{i, c_{i}}(r)^{2} d r\right]$ are finite when the $c_{i} s$ are normally distributed, and clearly $E\left[\int_{0}^{1} J_{i, c_{i}}(r) d W_{i}(r)\right]=0$. Thus, by the weak law of large numbers (WLLN), as $n \rightarrow \infty$,

$$
\begin{gathered}
\frac{1}{n} \sum_{i=1}^{n} \int_{0}^{1} J_{i, c_{i}}(r)^{2} d r \rightarrow p E\left[\int_{0}^{1} J_{i, c_{i}}(r)^{2} d r\right], \\
\frac{1}{n} \sum_{i=1}^{n} \int_{0}^{1} J_{i, c_{i}}(r) d W_{i}(r) \rightarrow_{p} 0
\end{gathered}
$$

and

$$
\frac{1}{n} \sum_{i=1}^{n} \eta_{i} \int_{0}^{1} J_{i, c_{i}}(r)^{2} d r \rightarrow_{p} E\left[\eta_{i} \int_{0}^{1} J_{i, c_{i}}(r)^{2} d r\right] .
$$


Combining these results, as $(T, n \rightarrow \infty)_{\text {seq }}$

$$
T(\hat{a}-a) \rightarrow_{p} E\left[\int_{0}^{1} J_{i, c_{i}}(r)^{2} d r\right]^{-1} E\left[\eta_{i} \int_{0}^{1} J_{i, c_{i}}(r)^{2} d r\right]
$$

Under the assumption of normally distributed $\eta_{i} s$, the two expectations in (7) can be calculated more explicitly. Using the properties of conditional expectations and the moment generating function (mgf) of the normal distribution, $M_{c_{i}}(t)=E\left[e^{t c_{i}}\right]=e^{c t+\sigma_{c}^{2} t^{2} / 2}$,

$$
\begin{aligned}
& E\left[\int_{0}^{1} J_{i, c_{i}}(r)^{2} d r\right] \\
= & \int_{0}^{1} \int_{0}^{r} E\left[e^{2(r-s) c_{i}}\right] d s d r \\
= & \frac{1}{8 \sigma_{c}^{3}}\left[2 \sigma_{c}\left(1-e^{2\left(c+\sigma_{c}^{2}\right)}\right)\right. \\
& \left.-\sqrt{2 \pi} e^{-\frac{c^{2}}{2 \sigma_{c}^{2}}}\left(c+2 \sigma_{c}^{2}\right)\left(\frac{1}{i} \Phi\left(i \frac{c}{\sqrt{2} \sigma_{c}}\right)-\frac{1}{i} \Phi\left(i \frac{c+2 \sigma^{2}}{\sqrt{2} \sigma}\right)\right)\right] \\
\equiv & \Psi_{1}\left(c, \sigma_{c}\right) .
\end{aligned}
$$

where $\Phi(x)=\frac{2}{\sqrt{\pi}} \int_{0}^{x} e^{-t^{2}} d t$ denotes the error function and $i=\sqrt{-1}$. Further,

$$
\begin{aligned}
& E\left[\eta_{i} \int_{0}^{1} J_{i, c_{i}}(r)^{2} d r\right] \\
= & \int_{0}^{1} \int_{0}^{r}\left(E\left[c_{i} e^{2(r-s) c_{i}}\right]-c E\left[e^{2(r-s) c_{i}}\right]\right) d s d r \\
= & \frac{1}{8 \sigma_{c}^{3}}\left[-2 \sigma_{c}\left(c-c e^{2\left(c+\sigma_{c}^{2}\right)}+2 \sigma_{c}^{2}\right)\right. \\
& \left.+\sqrt{2 \pi} e^{-\frac{c^{2}}{2 \sigma_{c}^{2}}}\left(c^{2}-\sigma_{c}^{2}+2 c \sigma_{c}^{2}\right)\left(\frac{1}{i} \Phi\left(i \frac{c}{\sqrt{2} \sigma}\right)-\frac{1}{i} \Phi\left(i \frac{c+2 \sigma^{2}}{\sqrt{2} \sigma}\right)\right)\right] \\
\equiv & \Psi_{2}\left(c, \sigma_{c}\right) .
\end{aligned}
$$

Thus, in sequential limits, as $(T, n \rightarrow \infty)_{\text {seq }}$,

$$
\hat{c}-c=T(\hat{a}-a) \rightarrow_{p} \Psi_{1}^{-1}\left(c, \sigma_{c}\right) \Psi_{2}\left(c, \sigma_{c}\right),
$$

and the pooled estimator, $\hat{c}$, provides inconsistent estimates of $c$. Define the function $f\left(c, \sigma_{c}\right)$ as the limit of $\hat{c}$,

$$
f\left(c, \sigma_{c}\right) \equiv c+\Psi_{1}^{-1}\left(c, \sigma_{c}\right) \Psi_{2}\left(c, \sigma_{c}\right) .
$$


Panel $\mathrm{A}$ in Table 1 gives the numerical values of the function $f\left(c, \sigma_{c}\right)$ for various combinations of $c$ and $\sigma_{c}$. It is readily apparent that the asymptotic bias of the pooled estimator for $c$ is already large for fairly small values of $\sigma_{c}$ and grows very large as $\sigma_{c}$ increases. Panel B in Table 1 shows the mean values of $\hat{c}$ from a Monte Carlo simulation with $n=100$ and $T=1,000$ using 10,000 repetitions. The distribution of the $c_{i} s$ is normal and the innovation processes, $\epsilon_{i, t}$, are also $i i d$ normal with $\sigma_{\epsilon}^{2}=1$. Figure 1 shows the density estimates of $\hat{c}$, from the same simulation exercise, for the cases where $c=-10,0,5$ and $\sigma_{c}=0,5,10$. The graphs clearly illustrate that the pooled estimator performs excellently for $\sigma_{c}=0$, but that its density starts drifting to the right as $\sigma_{c}$ increases. This is by any measure a large panel, and the $c_{i} s$ are drawn from a normal distribution, as was assumed when deriving the asymptotic limit function $f\left(c, \sigma_{c}\right)$.

By comparing the values in Panel A and Panel B in Table 1, it is obvious that the asymptotic limit of $\hat{c}$, given by $f\left(c, \sigma_{c}\right)$, provides a very poor approximation in finite samples as soon as the variance of the local-to-unity parameter starts to increase; the size of the sample in the Monte Carlo simulation was chosen to illustrate that this remains true also in very large samples. Two conclusions are thus immediate. First, the asymptotic limit of $\hat{c}$ given by $f\left(c, \sigma_{c}\right)$ cannot be used as a basis for a bias correcting procedure of $\hat{c}$ since it does not provide a good approximation in finite samples. Of course, even if $f\left(c, \sigma_{c}\right)$ did provide a good approximation, any bias correction scheme based on it would be complicated by the fact that $\sigma_{c}$ is unknown. Second, the pooled estimator works very poorly as soon as there is any variance, or heterogeneity, in the $c_{i} s$. Thus, applying the pooled estimator for $c$ to a panel, without any strong prior evidence or theory that the $c_{i} s$ are nearly identical, could lead to seriously biased inference.

How does one explain the poor finite sample performance of the asymptotic bias function? Observe that the actual finite sample bias is typically much smaller than the asymptotic bias, as $\sigma_{c}$ grows large. However, the gap between the asymptotic results and the finite sample results is not merely a function of the standard deviation, $\sigma_{c}$. For smaller values of $c$, a larger standard deviation is needed before the asymptotic value deviates substantially from the finite sample result. In fact, for large negative values of $c$, there is a very sharp increase in the asymptotic bias after $\sigma_{c}$ exceeds some value. For example, for $c=-50$, the asymptotic limit for $\sigma_{c}=7$ is equal to -48.8 , and for $\sigma_{c}=8$ the limit is 76.3 . Before this breakpoint, the finite sample results are similar to the asymptotic ones, but afterwards, they are vastly different. As $c$ becomes less negative, this effect becomes less distinct, and the growth of both the asymptotic bias and its deviation from the finite sample bias become smoother. 
Given these observations, a tentative explanation for the large difference between the asymptotic and finite sample results is the following. When $c_{i}>0$, the corresponding process is non-stationary and explosive. For positive $c_{i}$, the quantities $\eta_{i} \int_{0}^{1} J_{i, c_{i}}(r)^{2} d r$ and $\int_{0}^{1} J_{i, c_{i}}(r)^{2} d r$ will therefore grow very quickly in $c_{i}$. Thus, their mean values will be highly influenced by the tail behavior, or maximum value, of $c_{i}$. This causes no problems when calculating their analytical means, of course, but leads to problems when one tries to simulate them, which is essentially what is done in the Monte Carlo simulation. If the mean value depends on the tail behavior, it might be the case that extremely large sample sizes are needed before the simulated means approach the analytical ones. ${ }^{3}$ Since the functions $\eta_{i} \int_{0}^{1} J_{i, c_{i}}(r)^{2} d r$ and $\int_{0}^{1} J_{i, c_{i}}(r)^{2} d r$ do not grow fast in $c_{i}$ for $c_{i}$ non-positive, the above-mentioned problems only manifest themselves when there is a large enough probability for $c_{i}$ to be positive that it will significantly affect the mean. Otherwise, the tail behavior will have less of an impact on the mean. This would explain why a larger variance is needed for small $c_{i}$ before the gap between the finite sample value and the asymptotic value grows large. This also provides some intuition for the extremely large asymptotic biases from which the pooled estimator suffers.

The above reasoning suggests that the asymptotic bias approximation might perform better in cases where the support of the distribution of the local-to-unity parameters is bounded from above. To analyze this possibility, consider the case where the $c_{i} s$ are uniformly distributed on an interval $\left(c_{l}, c_{u}\right)$. In this case, $c=E\left[c_{i}\right]=\left(c_{u}+c_{l}\right) / 2$ and the asymptotic bias function of the pooled estimator can be written as

$$
f^{u}\left(c_{l}, c_{u}\right)=\frac{c_{u}+c_{l}}{2}+\Psi_{1}^{u}\left(c_{l}, c_{u}\right)^{-1} \Psi_{2}^{u}\left(c_{l}, c_{u}\right)
$$

where $\Psi_{1}^{u}\left(c_{l}, c_{u}\right) \equiv E\left[\eta_{i} \int_{0}^{1} J_{i, c_{i}}(r)^{2} d r\right]$ and $\Psi_{2}^{u}\left(c_{l}, c_{u}\right) \equiv E\left[\eta_{i} \int_{0}^{1} J_{i, c_{i}}(r)^{2} d r\right]$.

The numerical values for the function $f^{u}\left(c_{l}, c_{u}\right)$, obtained by using the mgf of the uniform distribution, are given in Panel A of Table 2. Panel B presents the corresponding mean pooled estimates of $c$ from a Monte Carlo simulation identical to the one described above, except that the local-to-unity parameters are now uniformly distributed. If the asymptotic limit function $f^{u}\left(c_{l}, c_{u}\right)$ provides a good approximation in finite samples, the corresponding values in Panel A and Panel B should be close. They are indeed much closer than in the normal case, and the asymptotic results do provide a good approximation to the finite sample values, lending some credibility to the explanation offered above. However, though the asymptotic results correspond better to the finite sample values in the uniform

\footnotetext{
${ }^{3}$ Steele (2001) gives an illustrative example of the problems of simulating tail probabilities. He argues that if one attempts to simulate the value of $E[1(x \geq 30)]$, where $x$ is standard normal, by naive methods, the number of simulations needs to be of an order greater than $10^{100}$.
} 
case, any inference method relying on these results would face the problem that the limit function $f^{u}\left(c_{l}, c_{u}\right)$ is not monotone in both $c_{l}$ and $c_{u}$ for all values of $c_{l}$ and $c_{u}$; the limit of $\hat{c}$ for $c_{u}=10$ is constant for all values of $c_{l}$ that are considered. Though this does appear to be a problem for large, positive $c_{u}$, it may not be relevant in practical applications, where $c_{u}$ is likely to be less than or equal to zero.

\section{A median based estimator}

\subsection{Bias properties}

Given the poor performance of the pooled estimator in the previous section, an alternative estimator is proposed in this section. Rather than summing up over the cross-section, consider applying the sample median instead. The intuition behind this approach is simple. The median is generally a more robust estimator than the mean and can perform better in cases where the mean performs poorly.

Let Assumption 1 hold, and let $\hat{\Lambda}_{i}$ and $\hat{\Omega}_{i}$ be consistent estimators, as $T \rightarrow \infty$, of $\Lambda_{i}$ and $\Omega_{i}$, respectively (see Moon and Phillips, 2000, for details). Begin with the inconsistent estimator of $c_{i}$,

$$
\tilde{c}_{i}=T\left(\tilde{a}_{i}-1\right)=\frac{\frac{1}{\hat{\Omega}_{i}}\left[\frac{1}{T}\left(\sum_{t=1}^{T} z_{i, t-1} z_{i, t}-T \hat{\Lambda}_{i}\right)-\frac{1}{T} \sum_{t=1}^{T} z_{i, t-1}^{2}\right]}{\frac{1}{\hat{\Omega}_{i}} \frac{1}{T^{2}} \sum_{t=1}^{T} z_{i, t-1}^{2}} \equiv \frac{m_{1, i, T}}{m_{2, i, T}}
$$

where $T \hat{\Lambda}_{i}$ is the serial correlation bias correction term and $m_{1, i, T}$ and $m_{2, i, T}$ are defined in the obvious manner. Define the median based estimator $\check{c}$ as follows,

$$
\check{c}=\frac{\operatorname{med}\left(m_{1, i, T}\right)}{\operatorname{med}\left(m_{2, i, T}\right)},
$$

where med $(\cdot)$ denotes the sample median. As $T \rightarrow \infty$, for fixed $i$,

$$
m_{1, i, T} \Rightarrow c_{i} \int_{0}^{1} J_{i, c_{i}}(r)^{2} d r+\int_{0}^{1} J_{i, c_{i}}(r) d W_{i}(r)=m_{1, i}
$$

and

$$
m_{2, i, T} \Rightarrow \int_{0}^{1} J_{i, c_{i}}(r)^{2} d r=m_{2, i}
$$

The division by $\hat{\Omega}_{i}$, in both the numerator and denominator in equation (13) enables the derivation of standardized results that are independent of the $\Omega_{i} s$; it is not necessary when the $\Omega_{i} s$ are identical 
for all $i$.

Define $\hat{\theta}_{k}, k=1,2$, as

$$
\hat{\theta}_{k}=\arg \min _{\theta_{k} \in \Theta_{k}} H_{k, n, T}\left(\theta_{k}\right)+O_{p}\left(\frac{1}{n}\right)=\arg \min _{\theta_{k} \in \Theta_{k}} \frac{1}{n} \sum_{i=1}^{n}\left|m_{k, i, T}-\theta_{k}\right|+O_{p}\left(\frac{1}{n}\right),
$$

and thus,

$$
\check{c}=\hat{\theta}_{1} / \hat{\theta}_{2} .
$$

Let $\theta_{1}^{0}$ and $\theta_{2}^{0}$ denote the medians of $m_{1, i}$ and $m_{2, i}$, so that

$$
\frac{1}{2}=\operatorname{Pr}\left(m_{1, i} \leq \theta_{1}^{0}\right) \text { and } \frac{1}{2}=\operatorname{Pr}\left(m_{2, i} \leq \theta_{2}^{0}\right)
$$

Assumption $3 \theta_{1}^{0}, \theta_{2}^{0} \in \Theta$, and $\Theta$ is a compact subset of $\mathbb{R}$.

Theorem 1 Under Assumptions 1 and 3, as $(n, T \rightarrow \infty)$,

$$
\hat{\theta}_{1} \rightarrow{ }_{p} \theta_{1}^{0}, \hat{\theta}_{2} \rightarrow{ }_{p} \theta_{2}^{0} \text {, and } \check{c}=\hat{\theta}_{1} / \hat{\theta}_{2} \rightarrow{ }_{p} \theta_{1}^{0} / \theta_{2}^{0} .
$$

So far, it has not been necessary to invoke Assumption 2; the above results hold for general distributions of the $c_{i} s$. However, in order to calculate $\theta_{1}^{0}$ and $\theta_{2}^{0}$, additional structure needs to be added to the problem. Analytical expressions for the medians of $m_{1, i}$ and $m_{2, i}$ are most likely not attainable, except for very special cases, but numerical results, given a distributional assumption on the $c_{i} s$, can be obtained. Therefore, I now make use of Assumption 2, and calculate numerical values for $\theta_{1}^{0}$ and $\theta_{2}^{0}$, for different combinations of $c$ and $\sigma_{c}$. The numerical methods used are described in the Appendix.

Panel $\mathrm{A}$ in Table 3 presents the numerical values of $\theta_{1}^{0} / \theta_{2}^{0}$, under Assumption 2, for various combinations of $c$ and $\sigma_{c}$. If $\check{c}$ were a consistent estimator of $c$, regardless of $\sigma_{c}$, all these values should equal their corresponding value for $c$. As is seen, this is not quite the case, but $\check{c}$ still turns out to have several desirable properties. First, for all combinations of $c$ and $\sigma_{c}$ recorded in Panel A of Table 3 , which arguably covers most empirically interesting cases, the bias is seen to be small and below 1.3 in absolute value. Indeed, for positive values of $c$, the bias is almost zero. Second, and just as importantly, for a fixed $c$, the bias varies only slightly with the variance parameter $\sigma_{c}$. The maximum difference observed, between $\sigma_{c}=0$ and $\sigma_{c}=10$, is no larger than 0.3 in absolute value, and is likely to be insignificant next to the variance of the estimates in any finite sample. This suggests that the 
same bias correction scheme can be used for a specific $c$, regardless of the value of $\sigma_{c}$. This is extremely convenient, since no estimate of $\sigma_{c}$ is then needed. Also, the bias correction is most naturally based on the case of $\sigma_{c}=0$, unless some specific prior information is available, for which the calculation of the bias is greatly simplified as compared to the case $\sigma_{c}>0$. Finally, for $\sigma_{c}=0$, the ratio $\theta_{1}^{0} / \theta_{2}^{0}$ is a monotone function of $c$, making bias correction feasible.

Given the experience with the pooled estimator, one would naturally wish to evaluate the correspondence between the asymptotic results presented in Panel A in Table 3 and the finite sample properties of the estimator. Panel B in Table 3 shows the results from a Monte Carlo study with a relatively large panel. The setup is the same as that used in the pooled case. Each simulated panel consists of $n=100$ time series, with $T=1,000$ observations in each. The innovation processes are normal $i i d(0,1)$ and the $c_{i} s$ are normally distributed. 10, 000 repetitions were performed and the mean values of the estimates are reported in Panel B of Table 3, for each combination of $c$ and $\sigma_{c}$. The estimates have not been bias corrected in any way, and the serial correlation correction term of the estimator is not included. Since the error terms all have the same variance, the division by $\hat{\Omega}_{i}$ in the numerator and denominator is not performed either.

If the asymptotic results are valid finite sample approximations, the values in Panel A and Panel $\mathrm{B}$ of Table 3 should be close for corresponding values of $c$ and $\sigma_{c}$. This also turns out to be the case, and the median estimator does appear to be robust with regard to the variance of the local-to-unity parameter.

Since the asymptotic bias seems like a reasonable approximation of the finite sample bias, a simple bias-correction scheme, based on the asymptotic results, can be implemented. Denote $g(c)=\theta_{1}^{0} / \theta_{2}^{0}$ for $\sigma_{c}=0$. Table 4 tabulates the values of $g(c)$ for $c \in[-50,10]$. As is seen, $g(c)$ is strictly increasing in $c$. A bias corrected version of $\check{c}$, which we will denote $\check{c}^{+}$, is now obtained by setting $\check{c}^{+}=g^{-1}(\check{c})$. The estimator $\check{c}^{+}$is now a nearly consistent estimator of $c$, in the general case of $\sigma_{c}>0$, and exactly consistent for the special case of $\sigma_{c}=0$. The bias correction scheme is particularly simple for the cases of $\theta_{1}^{0} / \theta_{2}^{0} \leq-8.78$ and for $\theta_{1}^{0} / \theta_{2}^{0} \geq 4.90$. According to the results of Table $4, \theta_{1}^{0} / \theta_{2}^{0}=c-1.28$ for $\theta_{1}^{0} / \theta_{2}^{0} \leq-8.78$ and $\theta_{1}^{0} / \theta_{2}^{0}=c$ for $\theta_{1}^{0} / \theta_{2}^{0} \geq 4.90$.

Performing an identical Monte Carlo simulation as the one described above, the bias corrected estimates $\check{c}^{+}$are calculated and the estimated densities of these estimates are plotted in Figure 2 . The densities of the estimates are centered very close to the true value of $c$, even for large values of $\sigma_{c}$.

Panels encountered in empirical practice are seldom as large as the ones used in the Monte Carlo 
simulation above. In Table 5 and Figure 3, I show the results from a Monte Carlo simulation with $n=20$ and $T=100$. The local-to-unity parameters are once again drawn from normal distributions, and the innovation processes are also iid normal, with unit variance. The mean values of the estimates presented in Table 5 generally look good. Considering the estimated densities, shown in Figure 3, the dispersion of the estimates for large values of $\sigma_{c}$ is, of course, fairly large, given the small sample size. But, for reasonable values, like $\sigma_{c}=5$ and $c=-10$, the estimator still appears to perform acceptably, given the sample size.

Simulation results not reported in this paper also illustrate that the estimator $\check{c}^{+}$works well for estimating average local-to-unity parameters when the distribution of the $c_{i} s$ is not normal. In the two cases where the $c_{i} s$ were drawn from either uniform distributions or Cauchy distributions, the estimator $\check{c}^{+}$was shown to deliver nearly unbiased estimates in finite samples. These results are available from the author upon request.

\subsection{Asymptotic normality and standard error estimation}

Having established convergence of $\check{c}$ to $\theta_{1}^{0} / \theta_{2}^{0}$ as $(n, T \rightarrow \infty)$, I now derive the asymptotic distribution of the estimator. Since the bias corrected estimator $\check{c}^{+}$is merely a shifted version of $\check{c}$, it will have the same asymptotic variance, but its distribution will be centered on $g^{-1}\left(\theta_{1}^{0} / \theta_{2}^{0}\right)$, rather than on $\theta_{1}^{0} / \theta_{2}^{0} \cdot$

Theorem 2 Under Assumptions 1-3, as $(T, n \rightarrow \infty)_{\text {seq }}$,

$$
\begin{aligned}
& \sqrt{n}\left(\check{c}-\theta_{1}^{0} / \theta_{2}^{0}\right) \\
\Rightarrow & N\left(0, \frac{1}{4\left(\theta_{1}^{0}\right)^{2} f_{1}\left(\theta_{1}^{0}\right)^{2}}+\frac{\left(\theta_{1}^{0}\right)^{2}}{4\left(\theta_{2}^{0}\right)^{4} f_{2}\left(\theta_{2}^{0}\right)^{2}}-\frac{v_{12}\left(\theta_{1}^{0}, \theta_{2}^{0}\right)}{2\left(\theta_{2}^{0}\right)^{2} f_{1}\left(\theta_{1}^{0}\right) f_{2}\left(\theta_{2}^{0}\right)}\right) .
\end{aligned}
$$

where $f_{k}\left(\theta_{k}\right)=\frac{d}{d \theta_{k}} F_{k}\left(\theta_{k}\right), F_{k}(\cdot)$ is the cumulative distribution function for $m_{k, i}$ for $k=1,2$, and

$$
v_{12}\left(\theta_{1}, \theta_{2}\right)=E\left[\operatorname{sign}\left(m_{1, i}-\theta_{1}\right) \operatorname{sign}\left(m_{2, i}-\theta_{2}\right)\right]
$$

In order to perform inference on $\check{c}$ and $\check{c}^{+}$, an estimate of the limit variance, given in equation (21), is needed. If one is willing to work with a specific parametric distribution for the $c_{i} s$, such as

\footnotetext{
${ }^{4}$ The asymptotic normality of the estimator is only shown for sequential limits. Subject to some additional rate restrictions on $n$ and $T$ the result also likely holds in joint limits as $(n, T \rightarrow \infty)$. However, due to the non-linear nature of the median operator, the proof for joint limits becomes very technical and is not crucial to the relatively applied discussion of this paper.
} 
the normal distribution, then the densities $f_{1}(\cdot)$ and $f_{2}(\cdot)$ can be calculated numerically for given $\theta_{1}$ and $\theta_{2}$, and estimates of $f_{1}\left(\theta_{1}^{0}\right)$ and $f_{2}\left(\theta_{2}^{0}\right)$ are given by numerical calculation of $f_{1}\left(\hat{\theta}_{1}\right)$ and $f_{2}\left(\hat{\theta}_{2}\right)$. Similarly, the expectation $v_{12}\left(\theta_{1}^{0}, \theta_{2}^{0}\right)$ could be numerically calculated. These numerical calculations are straightforward extensions of the methods used for finding the medians of $m_{1}$ and $m_{2}$, and will not be detailed here.

However, by using non-parametric methods, estimates of the desired quantities can be obtained without making any distributional assumptions. As argued above,

$$
F_{k, T}\left(\theta_{k}\right)=\operatorname{Pr}\left(m_{k, i, T} \leq \theta_{k}\right) \rightarrow \operatorname{Pr}\left(m_{k, i} \leq \theta_{k}\right)=F_{k}\left(\theta_{k}\right),
$$

as $T \rightarrow \infty$, for $k=1,2$ and $i=1, \ldots, n$. Since $F_{k}(\cdot)$ is continuously differentiable with a continuous derivative $f_{k}(\cdot)$, it follows that

$$
f_{k, T}\left(\theta_{k}\right)=\frac{d}{d \theta_{k}} F_{k, T}\left(\theta_{k}\right) \rightarrow \frac{d}{d \theta_{k}} F_{k}\left(\theta_{k}\right)=f_{k}\left(\theta_{k}\right)
$$

as $T \rightarrow \infty$. A consistent estimator for $f_{k}\left(\theta_{k}\right)$ is given by the kernel density estimator,

$$
\hat{f}_{k}\left(\theta_{k}\right)=\frac{1}{n h} \sum_{i=1}^{n} K\left(\frac{m_{k, i, T}-\theta_{k}}{h}\right)
$$

where $K(\cdot)$ is a kernel function and $h$ is the bandwidth parameter. Consistent estimates of $f_{1}\left(\theta_{1}^{0}\right)$ and $f_{2}\left(\theta_{2}^{0}\right)$ are now given by $\hat{f}_{1}\left(\hat{\theta}_{1}\right)$ and $\hat{f}_{2}\left(\hat{\theta}_{2}\right)$. Since the $m_{k, i, T} s$ are $i i d$, standard results for consistency of $\hat{f}_{k}\left(\theta_{k}\right)$ apply (e.g. Pagan and Ullah, 1999).

Finally, a consistent estimator of $v_{12}\left(\theta_{1}, \theta_{2}\right)$ is given by

$$
\hat{v}_{12}\left(\theta_{1}, \theta_{2}\right)=\frac{1}{n} \sum_{i=1}^{n} \operatorname{sign}\left(m_{1, i, T}-\theta_{1}\right) \operatorname{sign}\left(m_{2, i, T}-\theta_{2}\right)
$$

and a consistent estimate of $v_{12}\left(\theta_{1}^{0}, \theta_{2}^{0}\right)$ is provided by $\hat{v}_{12}\left(\hat{\theta}_{1}, \hat{\theta}_{2}\right)$.

The non-parametric approach is obviously more robust than the parametric one first described and is recommended in general. It is also the analogue of estimation procedures of the limiting covariance matrix in standard Least Absolute Deviations (LAD) regressions. 


\section{Conclusion}

In this paper, I analyze the problem of estimating the average local-to-unity parameter from a panel data set, where the local-to-unity parameters are treated as random variables. It is shown that the generalization from the setup with identical local-to-unity parameters raises some real issues in terms of consistency.

The pooled estimator for the average local-to-unity parameter is severely biased for even moderate variations in the local-to-unity parameters and could provide very misleading results if used indiscriminately. An alternative median based estimator is proposed instead. The idea behind this estimator is simple. To obtain more robust estimates than those provided by the pooled estimator, the sample median rather than the sample mean is used to extract the crucial cross-sectional information needed to estimate the local-to-unity parameter. The median based estimator is analyzed for the specific case of normally distributed local-to-unity parameters and is shown to exhibit a small asymptotic bias. The bias, however, is almost independent of the variance of the local-to-unity parameters and a simple bias-correction procedure is used to obtain nearly consistent estimates. The estimator is shown to work well in finite samples and appears robust against deviations from the normality assumption.

One issue not considered in this paper is that of heterogenous deterministic trends. Moon and Phillips (1999, 2000, and 2004) show that in the case of identical local-to-unity parameters, heterogenous trends cause the standard pooled estimator to become inconsistent. The effect of deterministic trends on the properties of the median based estimator proposed in this paper is left for future research.

\section{A Appendix}

\section{A.1 Numerical calculation of the medians of $m_{1, i}$ and $m_{2, i}$}

First, note that

$$
J_{i, c_{i}}(1)^{2}=1+2 c_{i} \int_{0}^{1} J_{i, c_{i}}(r)^{2} d r+2 \int_{0}^{1} J_{i, c_{i}}(r) d W_{i}(r)
$$

and, thus,

$$
c_{i} \int_{0}^{1} J_{i, c_{i}}(r)^{2} d r+\int_{0}^{1} J_{i, c_{i}}(r) d W_{i}(r)=\frac{1}{2}\left(J_{i, c_{i}}(1)^{2}-1\right) .
$$

Further, from Phillips (1987),

$$
J_{i, c_{i}}(r)\left|c_{i}=N\left(0, \frac{1}{2 c_{i}}\left(e^{2 r c_{i}}-1\right)\right)\right| c_{i}=\sqrt{\frac{1}{2 c_{i}}\left(e^{2 r c_{i}}-1\right)} N(0,1) \mid c_{i} .
$$


Thus,

$$
\begin{aligned}
& \operatorname{Pr}\left(J_{i, c_{i}}(r)^{2} \leq x^{2}\right) \\
= & \int_{-\infty}^{\infty}\left[\operatorname{Pr}\left(-x \leq J_{i, c_{i}}(r) \leq x \mid c_{i}\right)\right] f_{c}\left(c_{i}\right) d c_{i} \\
= & \int_{-\infty}^{\infty}\left[2 \Phi\left(\frac{x}{\sqrt{\frac{1}{2 c_{i}}\left(e^{2 r c_{i}}-1\right)}}\right)-1\right] f_{c}\left(c_{i}\right) d c_{i} .
\end{aligned}
$$

The median of $m_{1, i}$, which we denote $\theta_{1}$, is the solution to

$$
\frac{1}{2}=\operatorname{Pr}\left(m_{1, i} \leq \theta_{1}\right)=\operatorname{Pr}\left(\frac{1}{2}\left(J_{i, c_{i}}(1)^{2}-1\right) \leq \theta_{1}\right)=\operatorname{Pr}\left(-\sqrt{2 \theta_{1}+1} \leq J_{i, c_{i}}(1)^{2} \leq \sqrt{2 \theta_{1}+1}\right)
$$

$\theta_{1}$ is obtained by numerically evaluating the integral (27) and finding the value $x$ which sets this integral equal to one half. The median is then given by $\theta_{1}=\left(x^{2}-1\right) / 2$.

In order to derive the median of $m_{2, i}$, I use the characteristic function approach. By a result in Tanaka (1996, chapter 4), the characteristic function of $m_{2, i}$, for a fixed $c_{i}$, is given by

$$
\phi_{m_{2} \mid c_{i}}(t)=E\left[e^{i t m_{2, i}} \mid c_{i}\right]=E\left[\exp \left\{i t \int_{0}^{1} J_{i, c_{i}}(r)^{2} d r\right\} \mid c_{i}\right]=\frac{e^{-c_{i} / 2}}{\sqrt{\cos \lambda\left(c_{i}, t\right)-c_{i} \frac{\sin \lambda\left(c_{i}, t\right)}{\lambda\left(c_{i}, t\right)}}} \mid c_{i},
$$

where $\lambda\left(c_{i}, t\right)=\sqrt{2 i t-c_{i}^{2}}$. By Lévy's inversion theorem for nonnegative random variables,

$$
\operatorname{Pr}\left(m_{2, i} \leq x \mid c_{i}\right)=\frac{1}{\pi} \int_{0}^{\infty} \operatorname{Re}\left[\frac{1-e^{-i t x}}{i t} \phi_{m_{2} \mid c_{i}}(t)\right] d t
$$

Thus, under the assumption of a random $c_{i}$,

$$
\operatorname{Pr}\left(m_{2, i} \leq x\right)=\int_{-\infty}^{\infty} \operatorname{Pr}\left(m_{2, i} \leq x \mid c_{i}\right) f_{c}\left(c_{i}\right) d c_{i}=\int_{-\infty}^{\infty} \frac{1}{\pi} \int_{0}^{\infty} \operatorname{Re}\left[\frac{1-e^{-i t x}}{i t} \phi_{m_{2} \mid c_{i}}(t)\right] d t f_{c}\left(c_{i}\right) d c_{i}
$$

This integral is evaluated numerically and $\theta_{2}$ is given by the solution to $\frac{1}{2}=\operatorname{Pr}\left(m_{2, i} \leq x\right)$.

\section{A.2 Proofs of Theorems}

Proof of Theorem 1. The first order condition for $H_{k, n, T}\left(\theta_{k}\right)$, is

$$
G_{k, n, T}\left(\theta_{1}\right)=\frac{1}{n} \sum_{i=1}^{n}\left[1\left\{m_{k, i, T}>\theta_{k}\right\}-1\left\{m_{k, i, T} \leq \theta_{k}\right\}\right]=O_{p}\left(\frac{1}{n}\right)
$$


For fixed $n$, as $T \rightarrow \infty$, by the continuous mapping theorem (CMT),

$$
G_{k, n, T}\left(\theta_{1}\right) \Rightarrow \frac{1}{n} \sum_{i=1}^{n}\left[1\left\{m_{k, i}>\theta_{k}\right\}-1\left\{m_{k, i} \leq \theta_{k}\right\}\right]=G_{k, n}\left(\theta_{k}\right)
$$

The population moment condition for $G_{k, n}\left(\theta_{k}\right)$ is

$$
0=G_{k}\left(\theta_{k}\right)=E\left[1\left\{m_{k, i}>\theta_{k}\right\}\right]-E\left[1\left\{m_{k, i} \leq \theta_{k}\right\}\right]=1-2 F_{k}\left(\theta_{k}\right)
$$

where $F_{k}(\cdot)$ is the cumulative distribution function for $m_{k, i}$. By definition, $G_{1}\left(\theta_{1}^{0}\right)=G_{2}\left(\theta_{2}^{0}\right)=0$.

To prove the uniform convergence of $G_{1, n, T}(\cdot)$, it is sufficient to show that

$\sup _{\theta_{1} \in \Theta_{1}}\left|\frac{1}{n} \sum_{i=1}^{n} 1\left\{m_{1, i, T} \leq \theta_{1}\right\}-\operatorname{Pr}\left(m_{1, i} \leq \theta_{1}\right)\right|=\sup _{\theta_{1} \in \Theta_{1}}\left|\frac{1}{n} \sum_{i=1}^{n} 1\left\{m_{1, i, T} \leq \theta_{1}\right\}-E\left[1\left\{m_{1, i} \leq \theta_{1}\right\}\right]\right| \rightarrow{ }_{p} 0$

uniformly in $\theta_{1}$, as $(n, T \rightarrow \infty)$. Consider, for a fixed $\theta_{1}$,

$$
1\left\{m_{1, i, T} \leq \theta_{1}\right\}=1\left\{\frac{1}{\hat{\Omega}_{i}} \frac{1}{T} \sum_{t=1}^{T}\left[\left(\beta_{i, 0}+y_{i, t-1}\right) \frac{c_{i}}{T} y_{i, t-1}+\left(\beta_{i, 0}+y_{i, t-1}\right) \epsilon_{i, t}-T \hat{\Lambda}_{i}\right] \leq \theta_{1}\right\}
$$

For fixed $i$, by the continuous mapping theorem for almost surely continuous functions,

$$
1\left\{m_{1, i, T} \leq \theta_{1}\right\} \Rightarrow 1\left\{m_{1, i} \leq \theta_{1}\right\}=1\left\{c_{i} \int_{0}^{1} J_{i, c_{i}}(r)^{2} d r+\int_{0}^{1} J_{i, c_{i}}(r) d W_{i}(r) \leq \theta_{1}\right\}
$$

as $T \rightarrow \infty$, since $\hat{\Lambda}_{i} \rightarrow p \Lambda_{i}$ and $\hat{\Omega}_{i} \rightarrow p \Omega_{i}$. If the conditions of Corollary 1 of Phillips and Moon (1999) are satisfied, it then follows that

$$
\frac{1}{n} \sum_{i=1}^{n} 1\left\{m_{1, i, T} \leq \theta_{1}\right\} \rightarrow_{p} E\left[1\left\{m_{1, i} \leq \theta_{1}\right\}\right]
$$

as $(n, T \rightarrow \infty)$ for fixed $\theta_{1}$. Since $1\left\{m_{1, i, T} \leq \theta_{1}\right\}$ is uniformly bounded, $\left\|1\left\{m_{1, i, T} \leq \theta_{1}\right\}\right\|$ is uniformly integrable in $T$ for all $i$ (Billingsley, 1995) and the other conditions of Corollary 1 of Phillips and Moon (1999) hold trivially. Pointwise convergence, for fixed $\theta_{1}$, as $(n, T \rightarrow \infty)$, is thus established.

Since $\Theta$ is a compact space, to establish uniform convergence one only needs to show that

$$
X_{1, n, T}\left(\theta_{1}\right)=\frac{1}{n} \sum_{i=1}^{n}\left(1\left\{m_{1, i, T} \leq \theta_{1}\right\}-E\left[1\left\{m_{1, i} \leq \theta_{1}\right\}\right]\right)
$$


is stochastically equicontinuous. This follows by standard arguments and the proof is not detailed here. The same arguments can be applied to $G_{2, n, T}(\cdot)$ and will not be repeated. Thus, as $(n, T \rightarrow \infty)$,

$$
G_{k, n, T}\left(\theta_{1}\right) \rightarrow{ }_{p} 1-2 \operatorname{Pr}\left(m_{k, i} \leq \theta_{k}\right)
$$

uniformly in $\theta_{k}$ and the desired result follows.

Proof of Theorem 2. Observe first, that for fixed $n$, as $T \rightarrow \infty$,

$$
\sqrt{n}\left(\begin{array}{c}
G_{1, n, T}\left(\theta_{1}^{0}\right) \\
G_{2, n, T}\left(\theta_{2}^{0}\right)
\end{array}\right) \Rightarrow \frac{1}{\sqrt{n}} \sum_{i=1}^{n}\left(\begin{array}{c}
{\left[1\left\{m_{1, i}>\theta_{1}^{0}\right\}-1\left\{m_{1, i} \leq \theta_{1}^{0}\right\}\right]} \\
{\left[1\left\{m_{2, i}>\theta_{2}^{0}\right\}-1\left\{m_{2, i} \leq \theta_{2}^{0}\right\}\right]}
\end{array}\right)
$$

By the Lindeberg-Feller central limit theorem (CLT), as $n \rightarrow \infty$,

$$
\frac{1}{\sqrt{n}} \sum_{i=1}^{n}\left(\begin{array}{c}
{\left[1\left\{m_{1, i}>\theta_{1}^{0}\right\}-1\left\{m_{1, i} \leq \theta_{1}^{0}\right\}\right]} \\
{\left[1\left\{m_{2, i}>\theta_{2}^{0}\right\}-1\left\{m_{2, i} \leq \theta_{2}^{0}\right\}\right]}
\end{array}\right) \Rightarrow N(0, V)
$$

where

$$
V=\left(\begin{array}{cc}
1 & E\left[\operatorname{sign}\left(m_{1, i}-\theta_{1}^{0}\right) \operatorname{sign}\left(m_{2, i}-\theta_{2}^{0}\right)\right] \\
E\left[\operatorname{sign}\left(m_{1, i}-\theta_{1}^{0}\right) \operatorname{sign}\left(m_{2, i}-\theta_{2}^{0}\right)\right] & 1
\end{array}\right) .
$$

Thus, as $(T, n \rightarrow \infty)_{\text {seq }}$,

$$
\sqrt{n}\left(\begin{array}{c}
G_{1, n, T}\left(\theta_{1}^{0}\right) \\
G_{2, n, T}\left(\theta_{2}^{0}\right)
\end{array}\right) \Rightarrow N(0, V)
$$

Next, for $k=1,2$, for fixed $n$, as $T \rightarrow \infty$,

$$
\nu_{k, n, T}(\theta)=\sqrt{n}\left(G_{k, n, T}(\theta)-G_{k}(\theta)\right) \Rightarrow \sqrt{n}\left(G_{k, n}(\theta)-G_{k}(\theta)\right) .
$$

By standard arguments for LAD estimators, $\sqrt{n}\left(G_{k, n}(\theta)-G_{k}(\theta)\right)$ is stochastically equicontious as $n \rightarrow \infty$. It follows that $\nu_{k, n, T}(\theta)$ is stochastically equicontious as $(T, n \rightarrow \infty)_{\text {seq }}$.

From the expressions of $F_{1}\left(\theta_{1}\right)$ and $F_{2}\left(\theta_{2}\right)$, derived in Appendix A.1, it is obvious that they are both differentiable, and, hence, so are $G_{1}\left(\theta_{1}\right)$ and $G_{2}\left(\theta_{2}\right)$. Having established the asymptotic normality of $G_{k, n, T}\left(\theta_{k}^{0}\right)$, and the stochastic equicontinuity of the normalized process $\nu_{k, n, T}(\theta)$, the asymptotic normality of $\left(\hat{\theta}_{1}, \hat{\theta}_{2}\right)^{\prime}$ now follows from standard results for extremum estimators with non- 
smooth criterion functions (e.g. Theorem 7.1. in Newey and Mcfadden, 1994). The limiting covariance matrix is given by

$$
\left(\begin{array}{c}
\frac{d}{d \theta^{\prime}} G_{1}\left(\theta_{1}\right) \\
\frac{d}{d \theta^{\prime}} G_{2}\left(\theta_{2}\right)
\end{array}\right)^{-1} V\left(\begin{array}{c}
\frac{d}{d \theta^{\prime}} G_{1}\left(\theta_{1}\right) \\
\frac{d}{d \theta^{\prime}} G_{2}\left(\theta_{2}\right)
\end{array}\right)^{-1}=\left(\begin{array}{cc}
\frac{1}{f_{1}\left(\theta_{1}\right)^{2}} & \frac{v_{12}\left(\theta_{1}, \theta_{2}\right)}{f_{1}\left(\theta_{1}\right) f_{2}\left(\theta_{2}\right)} \\
\frac{v_{12}\left(\theta_{1}, \theta_{2}\right)}{f_{1}\left(\theta_{1}\right) f_{2}\left(\theta_{2}\right)} & \frac{1}{f_{2}\left(\theta_{2}\right)^{2}}
\end{array}\right)
$$

The final result for $\hat{\theta}_{1} / \hat{\theta}_{2}$ folllows from the delta method. 


\section{References}

[1] Billingsley, P., 1995. Probability and Measure, Third Edition (Wiley, New York).

[2] Campbell, J., and M. Yogo, 2003. Efficient Tests of Stock Return Predictability, Working Paper, Harvard University.

[3] Cavanagh, C., G. Elliot, and J. Stock, 1995. Inference in models with nearly integrated regressors, Econometric Theory 11, 1131-1147.

[4] Choi, I., 2001. Unit Root Tests for Panel Data, Journal of International Money and Finance 20, 249-272.

[5] Elliot, G., T.J. Rothenberg, and J.H. Stock, 1996. Efficient Tests for an Autoregressive Unit Root, Econometrica 64, 813-836.

[6] Levin A., F. Lin, and C. Chu, 2002. Unit Root Tests in Panel Data: Asymptotic and Finite-Sample Properties, Journal of Econometrics 108, 1-24.

[7] Maddala, G.S., and S. Wu, 1999. A Comparative Study of Unit Root Tests with Panel Data and a New Simple Test, Oxford Bulletin of Economics and Statistics 61, 631-651.

[8] Moon H.R., and B. Perron, 2003. Testing for a Unit Root in Panels with Dynamic Factors, CLEO Working Paper, USC.

[9] Moon H.R., B. Perron, and P.C.B Phillips, 2003. Incidental Trends and the Power of Panel Unit Root Tests, Cowles Foundation Discussion Paper 1435.

[10] Moon, H.R., and P.C.B. Phillips, 1999. Maximum Likelihood Estimation in Panels with Incidental Trends, Oxford Bulletin of Economics and Statistics 61, 711-748.

[11] Moon, H.R., and P.C.B. Phillips, 2000. Estimation of Autoregressive Roots near Unity using Panel Data, Econometric Theory 16, 927-998.

[12] Moon, H.R., and P.C.B. Phillips, 2004. GMM Estimation of Autoregressive Roots Near Unity with Panel Data, Econometrica 72, 467-522.

[13] Newey, W.K., D. McFadden, 1994. Large sample estimation and hypothesis testing, in Engle, R.F., and D.L. McFadden, eds., Handbook of Econometric, Vol. IV (North-Holland, Amsterdam) 2111-2245.

[14] Pagan, A., and A. Ullah, 1999. Nonparametric Econometrics, Cambridge University Press.

[15] Phillips, P.C.B, 1987. Towards a Unified Asymptotic Theory of Autoregression, Biometrika 74, 535-547..

[16] Phillips, P.C.B., and H.R. Moon, 1999. Linear Regression Limit Theory for Nonstationary Panel Data, Econometrica 67, 1057-1111.

[17] Phillips, P.C.B., H.R. Moon, and Z. Xiao, 1998. How to estimate autoregressive roots near unity, Cowles Foundation Discussion Paper 1191.

[18] Phillips, P.C.B., and P. Perron, 1988. Testing for a Unit Root in Time Series Regression, Biometrika $75,335-346$.

[19] Steele, J.M., 2001. Stochastic Calculus and Financial Applications (Springer, New York).

[20] Tanaka, K., 1996. Time Series Analysis: Nonstationary and Noinvetible Distribution Theory (Wiley, New York).

[21] Quah, D., 1994. Exploiting Cross-Section Variations for Unit Root Inference in Dynamic Panels, Economics Letters 44, 9-19. 
Table 1: The bias properties of the pooled estimator in the case of normally distributed local-to-unity parameters. Panel A shows the numerical values for the limit function of the pooled estimator, $f\left(c, \sigma_{c}\right)$. Panel B shows the mean values of the pooled estimates of $c$, $\hat{c}$, from a Monte Carlo simulation with $n=100$ and $T=1,000$, using 10,000 repetitions. The innovations are iid normal with variance equal to one. The local-to-unity parameters are also drawn from normal distributions with mean $c$ given by the left most column and standard deviation $\sigma_{c}$ given by the top row.

\begin{tabular}{|c|c|c|c|c|c|c|c|c|c|c|c|}
\hline \multirow[b]{2}{*}{$c$} & \multicolumn{11}{|c|}{$\sigma_{c}$} \\
\hline & 0.0 & 1.0 & 2.0 & 3.0 & 4.0 & $\overline{5.0}$ & 6.0 & 7.0 & 8.0 & 9.0 & 10.0 \\
\hline \multicolumn{12}{|c|}{ Panel A. } \\
\hline-50.0 & -50.0 & -50.0 & -49.9 & -49.8 & -49.7 & -49.5 & -49.3 & -48.8 & 76.3 & 110.5 & 148.7 \\
\hline-40.0 & -40.0 & -40.0 & -39.9 & -39.8 & -39.6 & -39.4 & -39.1 & 56.2 & 86.5 & 120.7 & 158.7 \\
\hline-30.0 & -30.0 & -30.0 & -29.9 & -29.7 & -29.5 & -29.1 & 40.1 & 66.5 & 96.7 & 130.8 & 168.8 \\
\hline-20.0 & -20.0 & -20.0 & -19.8 & -19.5 & -19.1 & 29.0 & 50.6 & 76.7 & 106.8 & 140.9 & 178.9 \\
\hline-10.0 & -10.0 & -9.9 & -9.6 & -8.7 & 20.3 & 38.7 & 60.8 & 86.9 & 116.9 & 150.9 & 188.9 \\
\hline-9.0 & -9.0 & -8.9 & -8.5 & -6.6 & 21.4 & 39.7 & 61.8 & 87.9 & 117.9 & 151.9 & 189.9 \\
\hline-8.0 & -8.0 & -7.9 & -7.5 & -2.6 & 22.5 & 40.8 & 62.8 & 88.9 & 118.9 & 152.9 & 191.0 \\
\hline-7.0 & -7.0 & -6.9 & -6.4 & 3.8 & 23.6 & 41.8 & 63.9 & 89.9 & 119.9 & 153.9 & 192.0 \\
\hline-6.0 & -6.0 & -5.8 & -5.2 & 8.5 & 24.7 & 42.8 & 64.9 & 90.9 & 120.9 & 155.0 & 193.0 \\
\hline-5.0 & -5.0 & -4.8 & -3.8 & 10.9 & 25.7 & 43.8 & 65.9 & 91.9 & 122.0 & 156.0 & 194.0 \\
\hline-4.0 & -4.0 & -3.8 & -2.1 & 12.4 & 26.8 & 44.9 & 66.9 & 92.9 & 123.0 & 157.0 & 195.0 \\
\hline-3.0 & -3.0 & -2.7 & 0.2 & 13.6 & 27.8 & 45.9 & 67.9 & 94.0 & 124.0 & 158.0 & 196.0 \\
\hline-2.0 & -2.0 & -1.6 & 2.8 & 14.7 & 28.9 & 46.9 & 69.0 & 95.0 & 125.0 & 159.0 & 197.0 \\
\hline-1.0 & -1.0 & -0.4 & 5.0 & 15.8 & 29.9 & 48.0 & 70.0 & 96.0 & 126.0 & 160.0 & 198.0 \\
\hline 0.0 & 0.0 & 0.9 & 6.6 & 16.9 & 31.0 & 49.0 & 71.0 & 97.0 & 127.0 & 161.0 & 199.0 \\
\hline 1.0 & 1.0 & 2.2 & 7.9 & 18.0 & 32.0 & 50.0 & 72.0 & 98.0 & 128.0 & 162.0 & 200.0 \\
\hline 2.0 & 2.0 & 3.4 & 9.1 & 19.0 & 33.0 & 51.0 & 73.0 & 99.0 & 129.0 & 163.0 & 201.0 \\
\hline 3.0 & 3.0 & 4.5 & 10.2 & 20.1 & 34.1 & 52.0 & 74.0 & 100.0 & 130.0 & 164.0 & 202.0 \\
\hline 4.0 & 4.0 & 5.6 & 11.3 & 21.1 & 35.1 & 53.1 & 75.0 & 101.0 & 131.0 & 165.0 & 203.0 \\
\hline 5.0 & 5.0 & 6.7 & 12.3 & 22.2 & 36.1 & 54.1 & 76.1 & 102.0 & 132.0 & 166.0 & 204.0 \\
\hline \multicolumn{12}{|c|}{ Panel B. } \\
\hline-50.0 & -50.0 & -50.0 & -49.9 & -49.9 & -49.7 & -49.5 & -49.3 & -49.1 & -48.7 & -48.4 & -47.9 \\
\hline-40.0 & -40.0 & -40.0 & -39.9 & -39.8 & -39.6 & -39.4 & -39.1 & -38.8 & -38.3 & -37.8 & -37.1 \\
\hline-30.0 & -30.0 & -30.0 & -29.9 & -29.7 & -29.5 & -29.2 & -28.8 & -28.2 & -27.4 & -26.0 & -23.6 \\
\hline-20.0 & -20.0 & -20.0 & -19.8 & -19.6 & -19.2 & -18.6 & -17.5 & -15.3 & -11.1 & -5.5 & 0.3 \\
\hline-10.0 & -10.0 & -9.9 & -9.6 & -8.9 & -7.0 & -2.5 & 2.6 & 6.7 & 9.7 & 12.3 & 14.8 \\
\hline-9.0 & -9.0 & -8.9 & -8.6 & -7.7 & -5.1 & -0.2 & 4.6 & 8.0 & 10.7 & 13.3 & 16.0 \\
\hline-8.0 & -8.0 & -7.9 & -7.5 & -6.4 & -3.0 & 2.1 & 6.1 & 9.1 & 11.7 & 14.3 & 16.9 \\
\hline-7.0 & -7.0 & -6.9 & -6.4 & -4.8 & -0.7 & 3.9 & 7.5 & 10.2 & 12.8 & 15.4 & 17.9 \\
\hline-6.0 & -6.0 & -5.9 & -5.3 & -3.0 & 1.5 & 5.6 & 8.6 & 11.2 & 13.8 & 16.4 & 18.9 \\
\hline-5.0 & -5.0 & -4.8 & -4.0 & -1.1 & 3.5 & 6.9 & 9.7 & 12.2 & 14.8 & 17.3 & 19.7 \\
\hline-4.0 & -4.0 & -3.8 & -2.6 & 1.0 & 5.0 & 8.1 & 10.7 & 13.2 & 15.8 & 18.3 & 20.9 \\
\hline-3.0 & -3.0 & -2.7 & -1.1 & 2.9 & 6.3 & 9.1 & 11.7 & 14.2 & 16.9 & 19.4 & 21.8 \\
\hline-2.0 & -2.0 & -1.6 & 0.6 & 4.4 & 7.4 & 10.1 & 12.7 & 15.3 & 17.9 & 20.4 & 22.9 \\
\hline-1.0 & -1.0 & -0.4 & 2.3 & 5.7 & 8.5 & 11.1 & 13.7 & 16.3 & 18.8 & 21.4 & 23.9 \\
\hline 0.0 & 0.0 & 0.8 & 3.7 & 6.8 & 9.5 & 12.1 & 14.7 & 17.3 & 19.8 & 22.3 & 24.9 \\
\hline 1.0 & 1.0 & 2.0 & 5.0 & 7.9 & 10.6 & 13.2 & 15.7 & 18.3 & 20.8 & 23.4 & 25.8 \\
\hline 2.0 & 2.0 & 3.2 & 6.1 & 8.9 & 11.5 & 14.2 & 16.8 & 19.3 & 21.7 & 24.4 & 26.8 \\
\hline 3.0 & 3.0 & 4.3 & 7.1 & 9.9 & 12.6 & 15.2 & 17.8 & 20.3 & 22.8 & 25.3 & 27.9 \\
\hline 4.0 & 4.0 & 5.4 & 8.2 & 10.9 & 13.6 & 16.1 & 18.7 & 21.3 & 23.9 & 26.3 & 28.9 \\
\hline 5.0 & 5.0 & 6.4 & 9.2 & 11.9 & 14.6 & 17.2 & 19.7 & 22.2 & 24.8 & 27.3 & 29.8 \\
\hline
\end{tabular}


Table 2: The bias properties of the pooled estimator in the case of uniformly distributed local-to-unity parameters, where $c_{l}$ is given by the left most column and $c_{u}$ by the top row. Panel A shows the numerical values of the function $f^{u}\left(c_{l}, c_{u}\right)$. Panel $\mathrm{B}$ reports the mean values of the pooled estimates of $c$ from a Monte Carlo simulation with $n=100$ and $T=1,000$, using 10,000 repetitions. The localto-unity parameters are drawn from uniform distributions with parameters $c_{l}$ and $c_{u}$. The numbers in parentheses are the true values for $c$.

\begin{tabular}{|c|c|c|c|c|c|c|c|}
\hline \multirow[b]{2}{*}{$c_{l}$} & \multicolumn{7}{|c|}{$c_{u}$} \\
\hline & -20.0 & -15.0 & -10.0 & -5.0 & 0.0 & 5.0 & 10.0 \\
\hline \multicolumn{8}{|c|}{ Panel A. } \\
\hline-25.0 & \multirow[t]{7}{*}{$\begin{array}{l}-22.4 \\
\end{array}$} & -19.6 & $\begin{array}{l}-16.4 \\
\end{array}$ & -12.6 & -6.5 & 4.2 & 9.4 \\
\hline-20.0 & & \multirow[t]{6}{*}{-17.4} & -14.5 & -10.9 & -5.4 & 4.2 & 9.4 \\
\hline-15.0 & & & \multirow{5}{*}{-12.3} & -9.2 & -4.3 & 4.2 & 9.4 \\
\hline-10.0 & & & & \multirow[t]{4}{*}{-7.2} & -3.1 & 4.2 & 9.4 \\
\hline-5.0 & & & & & \multirow[t]{3}{*}{-1.8} & 4.3 & 9.4 \\
\hline 0.0 & & & & & & \multirow[t]{2}{*}{4.3} & 9.4 \\
\hline 5.0 & & & & & & & 9.4 \\
\hline \multicolumn{8}{|c|}{ Panel B. } \\
\hline \multirow[t]{2}{*}{-25.0} & \multirow{14}{*}{$\begin{array}{r}-22.4 \\
(-22.5)\end{array}$} & \multirow{14}{*}{$\begin{array}{r}-19.6 \\
(-20.0) \\
-17.4 \\
(-17.5)\end{array}$} & \multirow{6}{*}{$\begin{array}{r}-16.5 \\
(-17.5) \\
-14.5 \\
(-15.0) \\
-12.4 \\
(-12.5)\end{array}$} & ב-12.6 & -6.7 & 3.8 & 9.2 \\
\hline & & & & $(-15.0)$ & $(-12.5)$ & $(-10.0)$ & $(-7.5)$ \\
\hline \multirow[t]{2}{*}{-20.0} & & & & -11.0 & -5.6 & 3.9 & 9.2 \\
\hline & & & & $(-12.5)$ & $(-10.0)$ & $(-7.5)$ & $(-5.0)$ \\
\hline \multirow[t]{2}{*}{-15.0} & & & & -9.2 & -4.5 & 4.0 & 9.2 \\
\hline & & & & $(-10.0)$ & $(-7.5)$ & $(-5.0)$ & $(-2.5)$ \\
\hline \multirow[t]{2}{*}{-10.0} & & & & -7.3 & -3.2 & 4.1 & 9.3 \\
\hline & & & & $(-7.5)$ & $(-5.0)$ & $(-2.5)$ & $(0.0)$ \\
\hline \multirow[t]{2}{*}{-5.0} & & & & & -1.8 & 4.2 & 9.3 \\
\hline & & & & & $(-2.5)$ & $(0.0)$ & $(2.5)$ \\
\hline \multirow[t]{2}{*}{0.0} & & & & & & 4.3 & 9.4 \\
\hline & & & & & & $(2.5)$ & $(5.0)$ \\
\hline \multirow[t]{2}{*}{5.0} & & & & & & & 9.4 \\
\hline & & & & & & & $(7.5)$ \\
\hline
\end{tabular}


Table 3: The bias properties of the median based estimator for normally distributed local-to-unity parameters. Panel A shows numerical values for the limit function, $\theta_{1}^{0} / \theta_{2}^{0}$, for different combinations of $c$ and $\sigma_{c}$. Panel $\mathrm{B}$ shows mean values of the median based estimates of $c, \check{c}$, from a Monte Carlo simulation with $n=100$ and $T=1,000$, using 10,000 repetitions. The innovations are iid normal with variance equal to one. The local-to-unity parameters are also drawn from normal distributions with $c$ given by the left most column and $\sigma_{c}$ given by the top row. The estimates have not been bias corrected.

\begin{tabular}{|c|c|c|c|c|c|c|c|c|c|c|c|}
\hline \multirow[b]{2}{*}{$c$} & \multicolumn{11}{|c|}{$\sigma_{c}$} \\
\hline & 0.0 & 1.0 & 2.0 & 3.0 & 4.0 & 5.0 & 6.0 & 7.0 & 8.0 & 9.0 & 10.0 \\
\hline \multicolumn{12}{|c|}{ Panel A. } \\
\hline-50.0 & -51.3 & -51.3 & -51.3 & -51.3 & -51.3 & -51.3 & -51.3 & -51.3 & -51.3 & -51.3 & -51.3 \\
\hline-40.0 & -41.3 & -41.3 & -41.3 & -41.3 & -41.3 & -41.3 & -41.3 & -41.3 & -41.3 & -41.3 & -41.3 \\
\hline-30.0 & -31.3 & -31.3 & -31.3 & -31.3 & -31.3 & -31.3 & -31.3 & -31.3 & -31.3 & -31.3 & -31.3 \\
\hline-20.0 & -21.3 & -21.3 & -21.3 & -21.3 & -21.3 & -21.3 & -21.3 & -21.3 & -21.3 & -21.3 & -21.3 \\
\hline-10.0 & -11.3 & -11.3 & -11.3 & -11.3 & -11.3 & -11.3 & -11.2 & -11.2 & -11.2 & -11.2 & -11.2 \\
\hline-9.0 & -10.3 & -10.3 & -10.3 & -10.3 & -10.3 & -10.3 & -10.2 & -10.2 & -10.2 & -10.2 & -10.2 \\
\hline-8.0 & -9.3 & -9.3 & -9.3 & -9.3 & -9.3 & -9.2 & -9.2 & -9.2 & -9.2 & -9.2 & -9.2 \\
\hline-7.0 & -8.3 & -8.3 & -8.3 & -8.3 & -8.3 & -8.2 & -8.2 & -8.2 & -8.2 & -8.2 & -8.2 \\
\hline-6.0 & -7.3 & -7.3 & -7.3 & -7.3 & -7.2 & -7.2 & -7.2 & -7.2 & -7.2 & -7.1 & -7.1 \\
\hline-5.0 & -6.3 & -6.3 & -6.3 & -6.2 & -6.2 & -6.2 & -6.2 & -6.1 & -6.1 & -6.1 & -6.1 \\
\hline-4.0 & -5.3 & -5.3 & -5.2 & -5.2 & -5.2 & -5.2 & -5.1 & -5.1 & -5.1 & -5.1 & -5.1 \\
\hline-3.0 & -4.2 & -4.2 & -4.2 & -4.2 & -4.1 & -4.1 & -4.1 & -4.1 & -4.1 & -4.1 & -4.0 \\
\hline-2.0 & -3.2 & -3.2 & -3.2 & -3.1 & -3.1 & -3.1 & -3.1 & -3.0 & -3.0 & -3.0 & -3.0 \\
\hline-1.0 & -2.1 & -2.1 & -2.1 & -2.0 & -2.0 & -2.0 & -2.0 & -2.0 & -2.0 & -2.0 & -2.0 \\
\hline 0.0 & -0.9 & -0.9 & -0.9 & -0.9 & -0.9 & -0.9 & -0.9 & -0.9 & -0.9 & -0.9 & -0.9 \\
\hline 1.0 & 0.4 & 0.3 & 0.3 & 0.3 & 0.3 & 0.3 & 0.3 & 0.3 & 0.2 & 0.2 & 0.2 \\
\hline 2.0 & 1.7 & 1.6 & 1.5 & 1.5 & 1.5 & 1.4 & 1.4 & 1.4 & 1.4 & 1.4 & 1.4 \\
\hline 3.0 & 2.9 & 2.7 & 2.6 & 2.6 & 2.6 & 2.6 & 2.6 & 2.6 & 2.6 & 2.6 & 2.6 \\
\hline 4.0 & 4.0 & 3.8 & 3.7 & 3.7 & 3.7 & 3.7 & 3.7 & 3.7 & 3.7 & 3.7 & 3.7 \\
\hline 5.0 & 5.0 & 4.8 & 4.8 & 4.8 & 4.8 & 4.8 & 4.8 & 4.8 & 4.8 & 4.8 & 4.8 \\
\hline \multicolumn{12}{|c|}{ Panel B. } \\
\hline$\overline{-50.0}$ & $\overline{-51.0}$ & $\overline{-51.0}$ & $\overline{-751.0}$ & $\overline{-~}-51.0$ & $\bar{~}-51.0$ & $\overline{-51.0}$ & -51.0 & -51.0 & -51.0 & -50.9 & $\overline{-51.0}$ \\
\hline-40.0 & -41.0 & -41.0 & -41.0 & -41.0 & -41.0 & -41.0 & -41.0 & -41.0 & -41.0 & -41.0 & -41.0 \\
\hline-30.0 & -31.0 & -31.0 & -31.0 & -31.0 & -31.0 & -31.0 & -31.0 & -31.0 & -31.0 & -31.0 & -31.0 \\
\hline-20.0 & -21.1 & -21.1 & -21.1 & -21.1 & -21.0 & -21.1 & -21.0 & -21.0 & -21.0 & -21.0 & -21.0 \\
\hline-10.0 & -11.1 & -11.1 & -11.1 & -11.1 & -11.1 & -11.1 & -11.1 & -11.1 & -11.0 & -11.0 & -11.0 \\
\hline-9.0 & -10.2 & -10.1 & -10.1 & -10.1 & -10.1 & -10.1 & -10.1 & -10.1 & -10.0 & -10.0 & -10.0 \\
\hline-8.0 & -9.1 & -9.2 & -9.1 & -9.1 & -9.1 & -9.1 & -9.1 & -9.1 & -9.0 & -9.0 & -9.0 \\
\hline-7.0 & -8.3 & -8.2 & -8.2 & -8.1 & -8.1 & -8.1 & -8.1 & -8.1 & -8.0 & -8.0 & -8.0 \\
\hline-6.0 & -7.3 & -7.2 & -7.3 & -7.2 & -7.1 & -7.1 & -7.1 & -7.1 & -7.0 & -7.0 & -7.0 \\
\hline-5.0 & -6.3 & -6.2 & -6.3 & -6.2 & -6.1 & -6.1 & -6.1 & -6.0 & -6.1 & -6.0 & -6.0 \\
\hline-4.0 & -5.2 & -5.2 & -5.3 & -5.2 & -5.1 & -5.1 & -5.1 & -5.1 & -5.0 & -5.0 & -5.0 \\
\hline-3.0 & -4.2 & -4.2 & -4.3 & -4.1 & -4.1 & -4.1 & -4.1 & -4.1 & -4.0 & -4.0 & -4.0 \\
\hline-2.0 & -3.3 & -3.2 & -3.1 & -3.1 & -3.1 & -3.0 & -3.0 & -3.0 & -3.0 & -3.0 & -3.0 \\
\hline-1.0 & -2.1 & -2.1 & -2.1 & -2.0 & -2.0 & -2.0 & -2.0 & -2.0 & -1.9 & -1.9 & -1.9 \\
\hline 0.0 & -1.0 & -0.9 & -0.9 & -0.9 & -0.9 & -0.9 & -0.9 & -0.9 & -0.8 & -0.8 & -0.8 \\
\hline 1.0 & 0.4 & 0.3 & 0.3 & 0.3 & 0.3 & 0.3 & 0.3 & 0.3 & 0.3 & 0.3 & 0.3 \\
\hline 2.0 & 1.7 & 1.6 & 1.5 & 1.4 & 1.5 & 1.5 & 1.5 & 1.5 & 1.5 & 1.5 & 1.5 \\
\hline 3.0 & 2.9 & 2.7 & 2.7 & 2.7 & 2.7 & 2.7 & 2.7 & 2.7 & 2.7 & 2.7 & 2.7 \\
\hline 4.0 & 4.0 & 3.8 & 3.8 & 3.8 & 3.8 & 3.8 & 3.8 & 3.8 & 3.8 & 3.8 & 3.8 \\
\hline 5.0 & 5.0 & 4.8 & 4.8 & 4.8 & 4.8 & 4.8 & 4.8 & 4.9 & 4.9 & 4.9 & 4.9 \\
\hline
\end{tabular}


Table 4: Numerical values for the limit function of the median based estimator, $\theta_{1}^{0} / \theta_{2}^{0}$, for the homogenous case $\sigma_{c}=0$.

\begin{tabular}{|c|c|c|c|c|c|c|c|c|c|c|c|}
\hline$c$ & $\theta_{1}^{0} / \theta_{2}^{0}$ & $c$ & $\theta_{1}^{0} / \theta_{2}^{0}$ & $c$ & $\theta_{1}^{0} / \theta_{2}^{0}$ & $c$ & $\theta_{1}^{0} / \theta_{2}^{0}$ & $c$ & $\theta_{1}^{\mathrm{O}} / \theta_{2}^{\mathrm{O}}$ & $c$ & $\theta_{1}^{0} / \theta_{2}^{0}$ \\
\hline-50.00 & 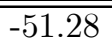 & -14.00 & -15.28 & $\overline{-26.80}$ & 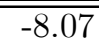 & $\overline{-23.20}$ & -4.45 & 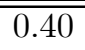 & $\overline{c-0.42}$ & $\bar{~} 4.00$ & 3.98 \\
\hline-49.00 & -50.28 & -13.00 & -14.28 & -6.70 & -7.97 & -3.10 & -4.35 & 0.50 & -0.28 & 4.10 & 4.08 \\
\hline-48.00 & -49.28 & -12.00 & -13.28 & -6.60 & -7.87 & -3.00 & -4.24 & 0.60 & -0.15 & 4.20 & 4.18 \\
\hline-47.00 & -48.28 & -11.00 & -12.28 & -6.50 & -7.77 & -2.90 & -4.14 & 0.70 & -0.01 & 4.30 & 4.29 \\
\hline-46.00 & -47.28 & -10.00 & -11.28 & -6.40 & -7.67 & -2.80 & -4.04 & 0.80 & 0.13 & 4.40 & 4.39 \\
\hline-45.00 & -46.28 & -9.90 & -11.18 & -6.30 & -7.57 & -2.70 & -3.94 & 0.90 & 0.27 & 4.50 & 4.49 \\
\hline-44.00 & -45.28 & -9.80 & -11.08 & -6.20 & -7.47 & -2.60 & -3.83 & 1.00 & 0.41 & 4.60 & 4.59 \\
\hline-43.00 & -44.28 & -9.70 & -10.98 & -6.10 & -7.37 & -2.50 & -3.73 & 1.10 & 0.54 & 4.70 & 4.69 \\
\hline-42.00 & -43.28 & -9.60 & -10.88 & -6.00 & -7.27 & -2.40 & -3.63 & 1.20 & 0.68 & 4.80 & 4.79 \\
\hline-41.00 & -42.28 & -9.50 & -10.78 & -5.90 & -7.17 & -2.30 & -3.52 & 1.30 & 0.82 & 4.90 & 4.90 \\
\hline-40.00 & -41.28 & -9.40 & -10.68 & -5.80 & -7.07 & -2.20 & -3.42 & 1.40 & 0.96 & 5.00 & 5.00 \\
\hline-39.00 & -40.28 & -9.30 & -10.58 & -5.70 & -6.97 & -2.10 & -3.32 & 1.50 & 1.09 & 6.00 & 6.00 \\
\hline-38.00 & -39.28 & -9.20 & -10.48 & -5.60 & -6.87 & -2.00 & -3.21 & 1.60 & 1.22 & 7.00 & 7.00 \\
\hline-37.00 & -38.28 & -9.10 & -10.38 & -5.50 & -6.77 & -1.90 & -3.11 & 1.70 & 1.36 & 8.00 & 8.00 \\
\hline-36.00 & -37.28 & -9.00 & -10.28 & -5.40 & -6.67 & -1.80 & -3.00 & 1.80 & 1.49 & 9.00 & 9.00 \\
\hline-35.00 & -36.28 & -8.90 & -10.18 & -5.30 & -6.57 & -1.70 & -2.89 & 1.90 & 1.61 & 10.00 & 10.00 \\
\hline-34.00 & -35.28 & -8.80 & -10.08 & -5.20 & -6.47 & -1.60 & -2.79 & 2.00 & 1.74 & & \\
\hline-33.00 & -34.28 & -8.70 & -9.98 & -5.10 & -6.37 & -1.50 & -2.68 & 2.10 & 1.87 & & \\
\hline-32.00 & -33.28 & -8.60 & -9.88 & -5.00 & -6.27 & -1.40 & -2.57 & 2.20 & 1.99 & & \\
\hline-31.00 & -32.28 & -8.50 & -9.78 & -4.90 & -6.17 & -1.30 & -2.46 & 2.30 & 2.11 & & \\
\hline-30.00 & -31.28 & -8.40 & -9.68 & -4.80 & -6.07 & -1.20 & -2.35 & 2.40 & 2.23 & & \\
\hline-29.00 & -30.28 & -8.30 & -9.58 & -4.70 & -5.97 & -1.10 & -2.24 & 2.50 & 2.35 & & \\
\hline-28.00 & -29.28 & -8.20 & -9.48 & -4.60 & -5.87 & -1.00 & -2.13 & 2.60 & 2.47 & & \\
\hline-27.00 & -28.28 & -8.10 & -9.38 & -4.50 & -5.76 & -0.90 & -2.02 & 2.70 & 2.58 & & \\
\hline-26.00 & -27.28 & -8.00 & -9.28 & -4.40 & -5.66 & -0.80 & -1.90 & 2.80 & 2.69 & & \\
\hline-25.00 & -26.28 & -7.90 & -9.18 & -4.30 & -5.56 & -0.70 & -1.79 & 2.90 & 2.81 & & \\
\hline-24.00 & -25.28 & -7.80 & -9.08 & -4.20 & -5.46 & -0.60 & -1.67 & 3.00 & 2.92 & & \\
\hline-23.00 & -24.28 & -7.70 & -8.98 & -4.10 & -5.36 & -0.50 & -1.55 & 3.10 & 3.03 & & \\
\hline-22.00 & -23.28 & -7.60 & -8.88 & -4.00 & -5.26 & -0.40 & -1.43 & 3.20 & 3.14 & & \\
\hline-21.00 & -22.28 & -7.50 & -8.78 & -3.90 & -5.16 & -0.30 & -1.31 & 3.30 & 3.24 & & \\
\hline-20.00 & -21.28 & -7.40 & -8.67 & -3.80 & -5.06 & -0.20 & -1.19 & 3.40 & 3.35 & & \\
\hline-19.00 & -20.28 & -7.30 & -8.57 & -3.70 & -4.96 & -0.10 & -1.06 & 3.50 & 3.46 & & \\
\hline-18.00 & -19.28 & -7.20 & -8.47 & -3.60 & -4.86 & 0.00 & -0.94 & 3.60 & 3.56 & & \\
\hline-17.00 & -18.28 & -7.10 & -8.37 & -3.50 & -4.75 & 0.10 & -0.81 & 3.70 & 3.67 & & \\
\hline-16.00 & -17.28 & -7.00 & -8.27 & -3.40 & -4.65 & 0.20 & -0.68 & 3.80 & 3.77 & & \\
\hline-15.00 & -16.28 & -6.90 & -8.17 & -3.30 & -4.55 & 0.30 & -0.55 & 3.90 & 3.88 & & \\
\hline
\end{tabular}


Table 5: Mean values of the bias corrected median estimates of $c, \check{c}^{+}$, from a Monte Carlo simulation with $n=20$ and $T=100$, using 10,000 repetitions. The innovations are iid normal with variance equal to one. The local-to-unity parameters are also drawn from a normal distribution with $c$ given by the left most column and $\sigma_{c}$ given by the top row.

\begin{tabular}{rrrrrrrrrrrr}
\hline \hline & \multicolumn{10}{c}{$\sigma_{c}$} \\
\cline { 2 - 11 } & 0.0 & 1.0 & 2.0 & 3.0 & 4.0 & 5.0 & 6.0 & 7.0 & 8.0 & 9.0 & 10.0 \\
\hline \hline-50.0 & -49.1 & -49.1 & -49.1 & -49.1 & -49.0 & -49.1 & -49.0 & -49.0 & -48.9 & -48.9 & -48.8 \\
-40.0 & -39.3 & -39.3 & -39.3 & -39.3 & -39.2 & -39.2 & -39.2 & -39.1 & -39.1 & -39.1 & -39.1 \\
-30.0 & -29.4 & -29.4 & -29.4 & -29.4 & -29.4 & -29.4 & -29.3 & -29.4 & -29.3 & -29.2 & -29.3 \\
-20.0 & -19.5 & -19.5 & -19.6 & -19.5 & -19.5 & -19.5 & -19.5 & -19.4 & -19.5 & -19.4 & -19.4 \\
-10.0 & -9.7 & -9.7 & -9.7 & -9.7 & -9.6 & -9.6 & -9.5 & -9.5 & -9.4 & -9.4 & -9.3 \\
-9.0 & -8.7 & -8.7 & -8.7 & -8.7 & -8.6 & -8.6 & -8.5 & -8.5 & -8.4 & -8.4 & -8.3 \\
-8.0 & -7.7 & -7.7 & -7.7 & -7.7 & -7.7 & -7.6 & -7.5 & -7.5 & -7.5 & -7.4 & -7.4 \\
-7.0 & -6.8 & -6.7 & -6.7 & -6.7 & -6.7 & -6.6 & -6.5 & -6.5 & -6.4 & -6.4 & -6.3 \\
-6.0 & -5.8 & -5.8 & -5.7 & -5.7 & -5.6 & -5.6 & -5.5 & -5.5 & -5.4 & -5.5 & -5.4 \\
-5.0 & -4.8 & -4.8 & -4.7 & -4.7 & -4.7 & -4.6 & -4.6 & -4.5 & -4.5 & -4.5 & -4.4 \\
-4.0 & -3.8 & -3.8 & -3.8 & -3.7 & -3.7 & -3.6 & -3.6 & -3.5 & -3.5 & -3.5 & -3.4 \\
-3.0 & -2.9 & -2.9 & -2.8 & -2.7 & -2.7 & -2.6 & -2.6 & -2.6 & -2.5 & -2.5 & -2.5 \\
-2.0 & -1.9 & -1.9 & -1.8 & -1.8 & -1.7 & -1.7 & -1.7 & -1.6 & -1.6 & -1.5 & -1.5 \\
-1.0 & -1.0 & -0.9 & -0.9 & -0.9 & -0.8 & -0.8 & -0.8 & -0.7 & -0.7 & -0.6 & -0.6 \\
0.0 & -0.0 & 0.0 & 0.0 & 0.1 & 0.1 & 0.1 & 0.2 & 0.3 & 0.3 & 0.4 & 0.5 \\
1.0 & 1.0 & 0.9 & 0.9 & 1.0 & 1.0 & 1.1 & 1.1 & 1.2 & 1.3 & 1.3 & 1.4 \\
2.0 & 2.0 & 1.9 & 1.9 & 1.9 & 2.0 & 2.0 & 2.1 & 2.2 & 2.2 & 2.3 & 2.4 \\
3.0 & 3.0 & 2.9 & 2.8 & 2.9 & 2.9 & 3.0 & 3.1 & 3.1 & 3.2 & 3.3 & 3.4 \\
4.0 & 4.0 & 3.8 & 3.8 & 3.9 & 3.9 & 4.0 & 4.1 & 4.2 & 4.3 & 4.3 & 4.3 \\
5.0 & 5.0 & 4.9 & 4.9 & 4.9 & 5.0 & 5.1 & 5.1 & 5.2 & 5.2 & 5.4 & 5.4 \\
\hline \hline
\end{tabular}


$\mathrm{c}=-10, \sigma_{\mathrm{c}}=0$

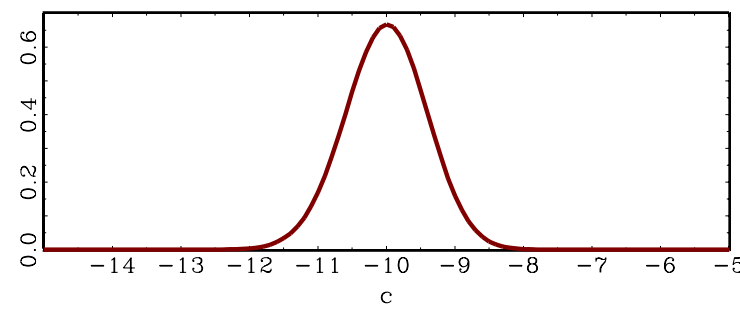

$\mathrm{c}=0, \sigma_{\mathrm{c}}=0$

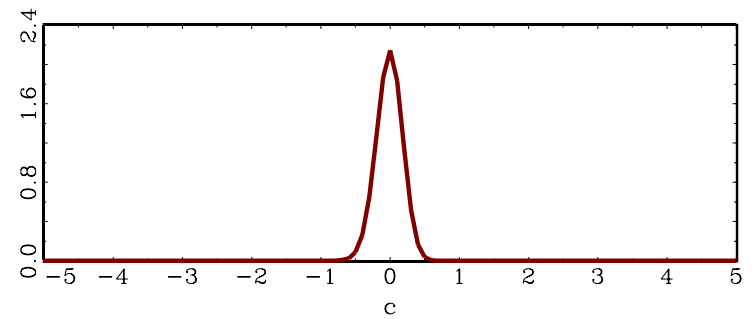

$c=5, \sigma_{c}=0$

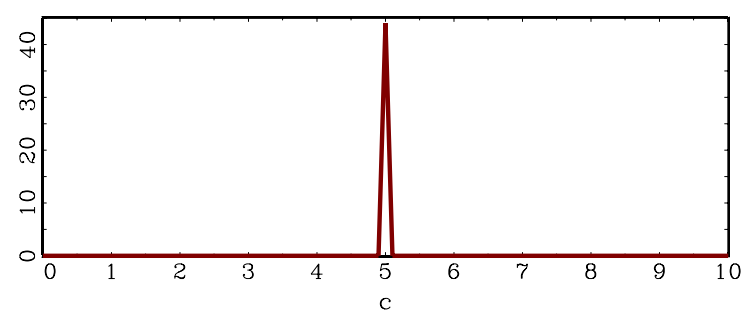

$\mathrm{c}=-10, \sigma_{\mathrm{c}}=5$ and $\sigma_{\mathrm{c}}=10$

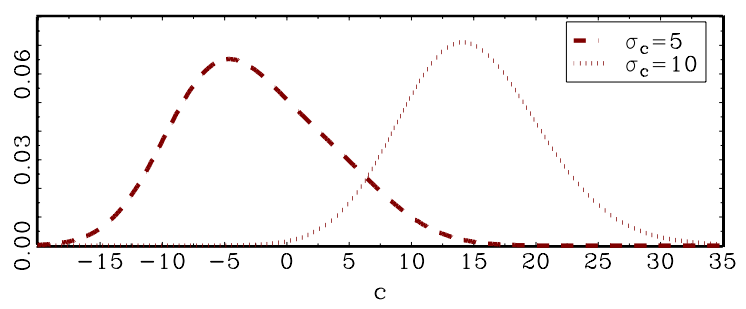

$c=0, \sigma_{c}=5$ and $\sigma_{c}=10$

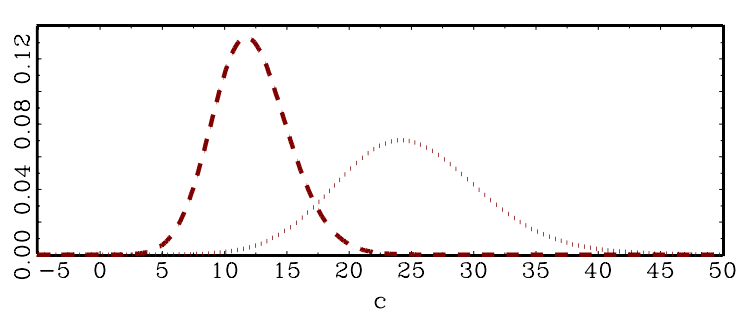

$c=5, \sigma_{c}=5$ and $\sigma_{c}=10$

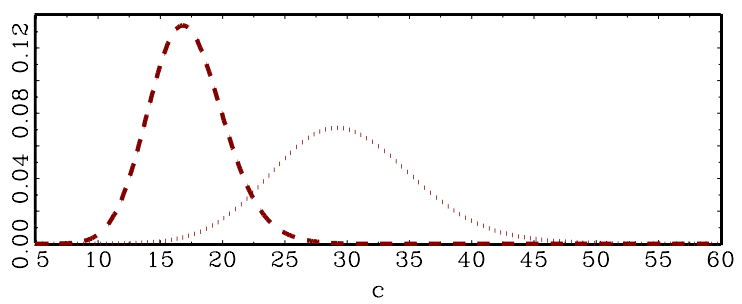

Figure 1: Estimates of the density functions of the pooled estimates of $c, \hat{c}$, in a Monte Carlo simulation. The sample size is $n=100$ and $T=1,000$, using 10,000 repetitions. The innovations are iid normal with variance equal to one. The local-to-unity parameters are also drawn from a normal distribution with the mean and variance given above each graph. In the right hand graphs, the dashed line corresponds to $\sigma_{c}=5$ and the dotted line to $\sigma_{c}=10$. 
$\mathrm{c}=-10, \sigma_{\mathrm{c}}=0$

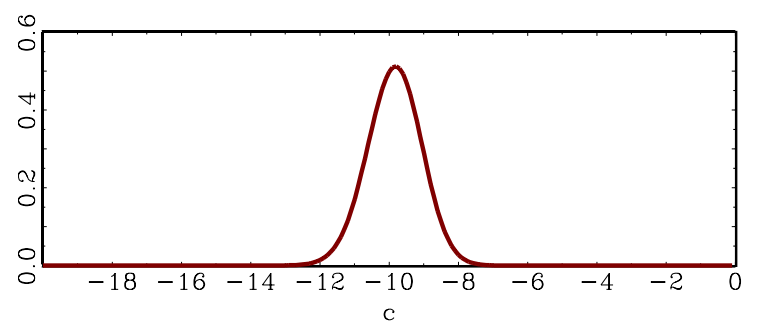

$c=0, \sigma_{c}=0$

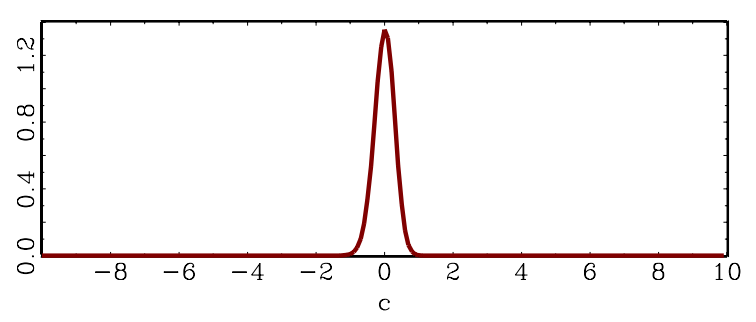

$c=5, \sigma_{c}=0$

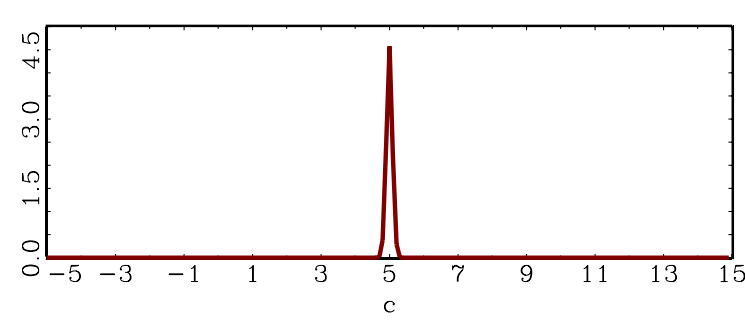

$\mathrm{c}=-10, \sigma_{c}=5$ and $\sigma_{c}=10$

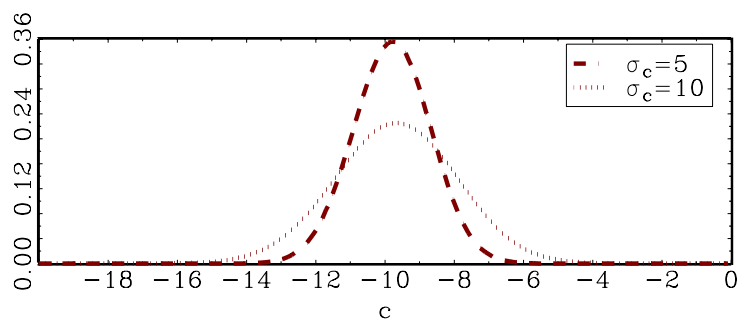

$c=0, \sigma_{c}=5$ and $\sigma_{c}=10$

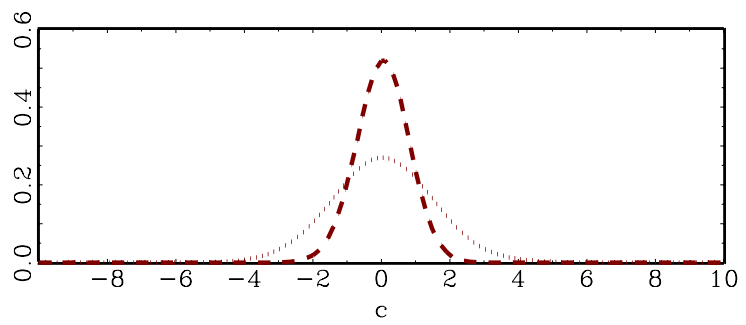

$c=5, \sigma_{c}=5$ and $\sigma_{c}=10$

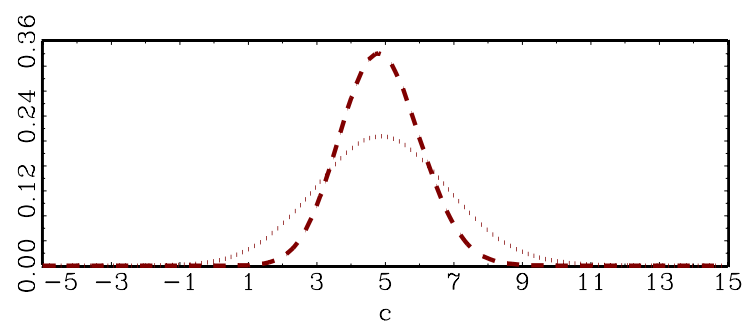

Figure 2: Estimates of the density functions of the bias corrected median estimates of $c, \check{c}^{+}$, in a Monte Carlo simulation. The sample size is $n=100$ and $T=1,000$, using 10,000 repetitions. The innovations are iid normal with variance equal to one. The local-to-unity parameters are also drawn from normal distributions with the mean and variance given above each graph. In the right hand graphs, the dashed line corresponds to $\sigma_{c}=5$ and the dotted line to $\sigma_{c}=10$. 

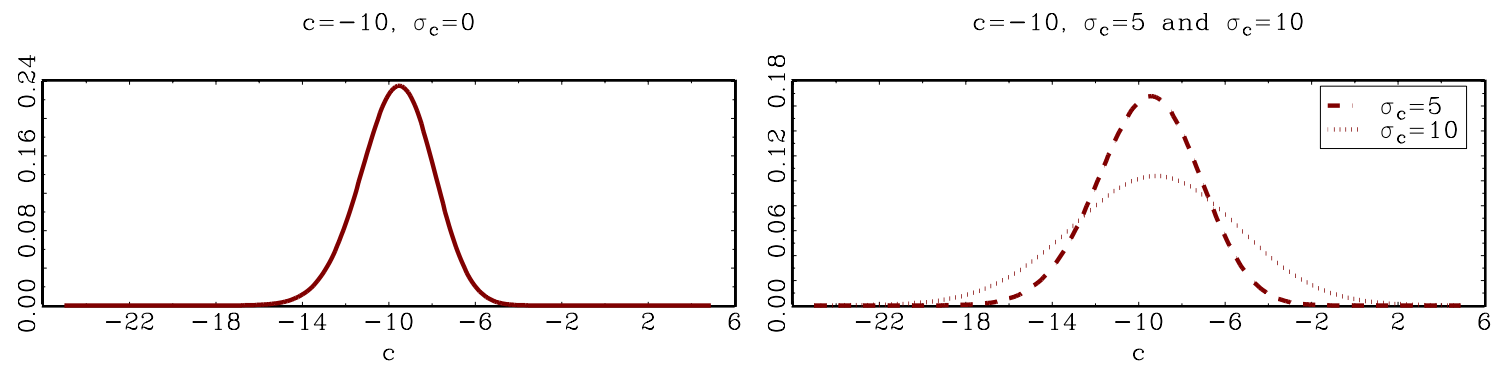

$\mathrm{c}=0, \sigma_{\mathrm{c}}=0$

$c=0, \sigma_{c}=5$ and $\sigma_{c}=10$
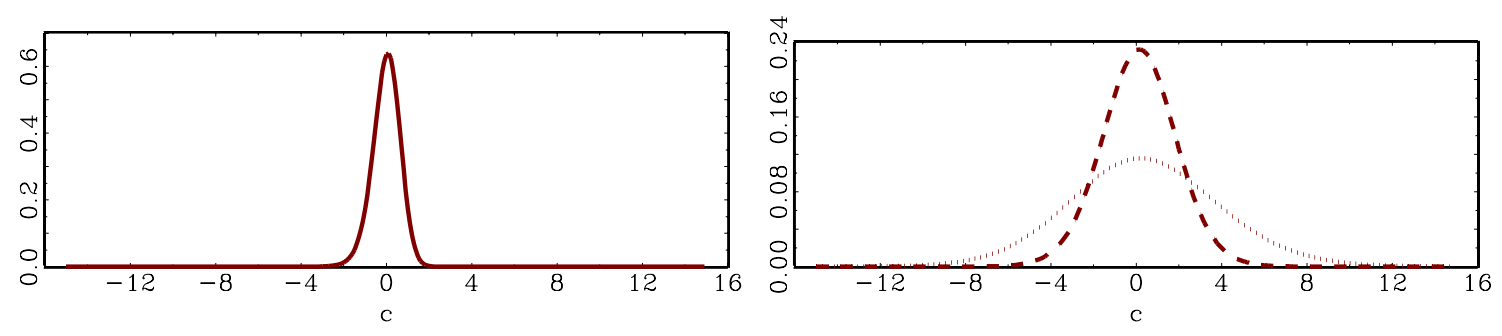

$c=5, \sigma_{c}=0$

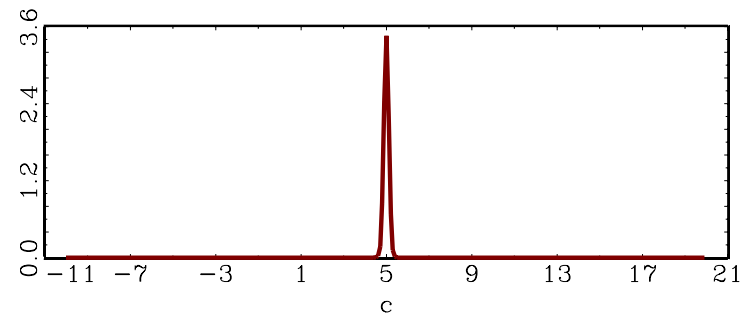

$c=5, \sigma_{c}=5$ and $\sigma_{c}=10$

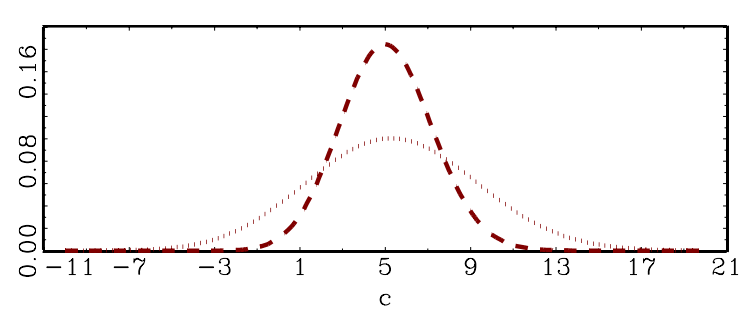

Figure 3: Estimates of the density functions of the bias corrected median estimates of $c, \check{c}^{+}$, in a Monte Carlo simulation. The sample size is $n=20$ and $T=100$, using 10,000 repetitions. The innovations are iid normal with variance equal to one. The local-to-unity parameters are also drawn from normal distributions with the mean and variance given above each graph. In the right hand graphs, the dashed line correspond to $\sigma_{c}=5$ and the dotted line to $\sigma_{c}=10$. 


\section{International Finance Discussion Papers}

IFDP

Number

Titles

Author(s)

2006

851

Exchange-Rate Pass-Through in the G-7 Countries

Jane E. Ihrig

Mario Marazzi

Alexander D.

Rothenberg

850

The Adjustment of Global External Imbalances: Does Partial

Exchange Rate Pass-Through to Trade Prices Matter?

Christopher Gust

Nathan Sheets

\section{5}

849

Interest Rate Rules, Endogenous Cycles and Chaotic Dynamics in Open Economies

Marco Airaudo

Luis-Felipe Zanna

848

Fighting Against Currency Depreciation Macroeconomic

Luis-Felipe Zanna Instability and Sudden Stops

The Baby Boom Predictability in House Prices and Interest Rates Robert F. Martin

846

Explaining the Global Pattern of Current Account Imbalances

Joseph W. Gruber

Steven B. Kamin

845

DSGE Models of High Exchange-Rate Volatility and Low Pass-Through

Giancarlo Corsetti

Luca Dedola

Sylvain Leduc

844

The Response of Global Equity Indexes to U.S. Monetary

Jon Wongswan Policy Announcements

Accounting Standards and Information: Inferences from

John Ammer Cross-Listed Financial Firms

Nathanael Clinton

Gregory P. Nini

842

Alternative Procedures for Estimating Vector Autoregressions Identified with Long-Run Restrictions

Lawrence J Christiano Martin Eichenbaum

Robert J. Vigfusson

Please address requests for copies to International Finance Discussion Papers, Publications, Stop 127, Board of Governors of the Federal Reserve System, Washington, DC 20551.

Email: publications-bog@ffrb.gov. Fax (202) 728-5886. 


\section{International Finance Discussion Papers}

IFDP

Number

841

840

839

838

837

836

835

834

833

832

831

830 $\underline{\text { Titles }}$

Monetary Policy and House Prices: A Cross-Country Study

International Capital Flows and U.S. Interest Rates

Effects of Financial Autarky and Integration: The Case of the South Africa Embargo

General-to-specific Modeling: An Overview and Selected Bibliography

Currency Crashes and Bond Yields in Industrial Countries

Estimating Elasticities for U.S. Trade in Services

SIGMA: A New Open Economy Model for Policy Analysis

Optimal Fiscal and Monetary Policy with Sticky Wages and Sticky Prices

Exchange Rate Pass-through to U.S. Import Prices: Some New Evidence

A Flexible Finite-Horizon Identification of Technology Shocks

Adjusting Chinese Bilateral Trade Data: How Big is China's Surplus

Order Flow and Exchange Rate Dynamics in Electronic Brokerage System Data $\underline{\text { Author(s) }}$

Alan G. Ahearne

John Ammer

Brian M. Doyle

Linda S. Kole

Robert F. Martin

Francis E. Warnock

Veronica C. Warnock

Brahima Coulibaly

Julia Campos

Neil R. Ericsson

David F. Hendry

Joseph E. Gagnon

Jaime Marquez

Christopher Erceg

Luca Guerrieri

Christopher Gust

Sanjay K. Chugh

Mario Marazzi

Nathan Sheets

Robert J. Vigufsson

And Others

Neville Francis

Michael T. Owyang

Jennifer E. Roush

John W. Schindler Dustin H. Beckett

David W. Berger Alain P. Chaboud Sergey V. Chernenko Edward Howorka

Raj S. Krishnasami

Iyer

David Liu

Jonathan H. Wright 